\title{
Running coupling in Yang-Mills theory: A flow equation study
}

\author{
Holger Gies* \\ CERN, Theory Division, CH-1211 Geneva 23, Switzerland
}

(Received 19 March 2002; published 1 July 2002)

\begin{abstract}
The effective average action of Yang-Mills theory is analyzed in the framework of exact renormalization group flow equations. Employing the background-field method and using a cutoff that is adjusted to the spectral flow, the running of the gauge coupling is obtained on all scales. In four dimensions and for the gauge groups $\mathrm{SU}(2)$ and $\mathrm{SU}(3)$, the coupling approaches a fixed point in the infrared.
\end{abstract}

DOI: 10.1103/PhysRevD.66.025006

PACS number(s): 11.10.Hi, 11.15.Tk

\section{INTRODUCTION AND SUMMARY}

Understanding the infrared sector of Yang-Mills theory still represents a challenge in quantum field theory. The strong coupling of the system and the rich dynamics of its degrees of freedom are well beyond the applicability of many field theoretic methods. Even without attempting to solve the theory at one fell swoop, it is already difficult to find (and then answer) questions that can be disentangled from the full complexity of the problem.

In this work we study Yang-Mills theory in the framework of renormalization group (RG) flow equations [1] for the effective average action [2], concentrating solely on the running gauge coupling. Whereas perturbation theory describes asymptotic freedom of the coupling in the high-energy limit, it fails to predict anything at low energies except for its own failure-manifested by the Landau pole singularity. Even without unveiling the complete infrared structure of gauge theories (including confinement and a mass gap), an analytic knowledge of the running of the coupling towards lower energies beyond perturbation theory is desirable. Exact RG flow equations represent an appropriate tool for tackling this problem.

Flow equation for the effective average action. Being a "coarse-grained" free-energy functional, the effective average action $\Gamma_{k}$ governs the dynamics of a theory at a momentum scale $k$. It comprises the effects of all quantum fluctuations of the dynamical field variables with momenta larger than $k$, whereas fluctuations with momenta smaller than $k$ have not (yet) been integrated out. Decreasing $k$ corresponds to integrating out more and more momentum shells of the quantum fluctuations. This successive averaging is implemented by a $k$-dependent infrared cutoff term $\Delta_{k} S$ which is added to the classical action in the standard Euclidean functional integral. This term gives a momentum-dependent mass square $R_{k}\left(p^{2}\right)$ to the field modes with momentum $p$ which vanishes for $p^{2} \gg k^{2}$. Regarding $\Gamma_{k}$ as a function of $k$, the effective average action runs along a RG trajectory in the space of all action functionals that interpolates between the classical action $S=\Gamma_{k \rightarrow \infty}$ and the conventional quantum effective action $\Gamma=\Gamma_{k \rightarrow 0}$. The response of $\Gamma_{k}$ to an infinitesimal variation of the scale $k$ is described by a functional dif-

*Email address: Holger.Gies@ @ern.ch ferential equation, the flow equation (an exact RG equation). In a symbolic notation,

$$
\partial_{t} \Gamma_{k}=\frac{1}{2} \operatorname{STr}\left[\partial_{t} R_{k}\left(\Gamma_{k}^{(2)}+R_{k}\right)^{-1}\right], \quad \partial_{t} \equiv k \frac{d}{d k},
$$

where $\Gamma_{k}^{(2)}$ denotes the second functional derivative of the effective average action with respect to the field variables and corresponds to the inverse exact propagator at the scale $k$.

Flow equation in gauge theories. The use of flow equations in gauge theories, as initiated in [3-5], is complicated by the fact that it is difficult to reconcile the Wilsonian idea of integrating out momentum shells of quantum fluctuations with gauge invariance. Working with gauge-noninvariant field variables such as gluons and ghosts, a regularization of the theory with a momentum cutoff necessarily breaks gauge invariance. Nevertheless, gauge-invariant flows can, in principle, be constructed by taking care of constraints imposed by the Ward identities which are modified by the presence of the cutoff $[4,6-8]$; in practice, resolving these constraints beyond perturbation theory is highly involved; for a review, see [9].

As an alternative, a formulation in terms of gaugeinvariant variables such as, for instance, Wilson loops may therefore be desirable and so has been proposed and worked out in [10]. Related to this, a gauge-invariant regularization has been formulated in [11] by constructing $\mathrm{SU}(N)$ YangMills theory from a spontaneously broken $\mathrm{SU}(N \mid N)$ supergauge extension; here the fermionic super-partners become massive and act as Pauli-Villars regulator fields without breaking the residual $\mathrm{SU}(N)$ gauge invariance. As a result, the one-loop $\beta$ function has been computed without any gauge fixing.

In this work we decide to employ the conventional and technically more feasible formulation in terms of the gluonic gauge field at the expense of only partially resolving the modified Ward identities resulting in less control over gauge invariance. In this way, we shall accept a compromise between calculational advantages and the implementation of complete quantum gauge invariance. In particular, we follow the strategy of [12], employing the background-field method. Our solution to the flow equation will be gauge invariant in the background field, but the renormalization group trajec- 
tory that connects the classical (bare) action with our quantum solution will not satisfy all requirements of gauge invariance (cf. Sec. II).

Truncations. Flow equations for interacting quantum field theories can be solved only approximately. A consistent and systematic approximation scheme is given by the method of truncations. Herein, the infinite space of all possible actions, spanned by the field operators compatible with the symmetries, is truncated to a subset of operators; the flow equation for the complete effective action can then be boiled down to the flow equations of the coefficients of these operators (generalized couplings). The renormalization trajectory in the space of all actions is thereby projected onto the hypersurface spanned by all operators of the truncation. For a selected truncation to be able to describe the physics of the system, its operators have to cover the dynamics of the relevant degrees of freedom of the system under consideration. Since the relevant degrees of freedom in strongly coupled quantum field theories, such as Yang-Mills theories, may change under the renormalization flow, a careful and deliberate choice of the truncation is halfway to the solution of the theory. In view of the many proposals concerning the "true" degrees of freedom in the infrared sector of Yang-Mills theory, their systematic study within a flow equation approach would be desirable. Along this direction, interesting and promising results have been obtained in [13] and [14], where the choices of the truncation have been based on the monopole picture of infrared Yang-Mills theory.

In the present work we follow a different strategy: we stick to the "gluonic language" and maintain the gauge field as the basic variable. This avoids complications inherent in the change of quantum variables, which has to be performed with great care (see, e.g., [15] and [16]). But in order to account for the fact that the "true" infrared degrees of freedom may have a complicated gluonic description, we include infinitely many gluonic invariants in our truncation; to be explicit, we consider a truncation in which the gaugeinvariant part of the effective action is an arbitrary function $W_{k}$ of the square of the field strength $F$,

$$
\Gamma_{k}^{\mathrm{inv}}[A]=\int W_{k}(\theta), \quad \theta:=\frac{1}{4} F_{\mu \nu}^{a} F_{\mu \nu}^{a},
$$

and the running of the coupling will be extracted from the flow of the linear $F_{\mu \nu}^{a} F_{\mu \nu}^{a}$ term in $W_{k}$, as it is standard in continuum quantum Yang-Mills theory. At weak coupling, it may be sufficient to approximate $W_{k}[\theta]$ by a finite series, i.e., a polynomial in $\theta$, being justifiable by simple power counting (higher operators are suppressed by powers of the ultraviolet cutoff). But at strong coupling, those higher operators can acquire large anomalous dimensions that completely obstruct a naive power-counting analysis. In fact, our results show that the flow of the complete function $W_{k}$ contributes to the running gauge coupling, and that the flow of higher order operators must not be neglected.

Beyond the approximations involved (i) in choosing Eq. (2) as our truncation (and neglecting other invariants) and (ii) in resolving the modified Ward identity only partially, we make a third approximation (iii) by neglecting any nontrivial running in the ghost and gauge-fixing sectors.

Regulators. For an explicit evaluation of the flow equation, a cutoff function (or regulator) $R_{k}$ has to be specified. This cutoff function is to some extent arbitrary (see Appendix D). In the denominator of the flow equation (1), it acts as an infrared cutoff for modes with momenta smaller than $k$; its derivative $\partial_{t} R_{k}$ in the numerator is peaked $\delta$-like around $k$ and thus implements the Wilsonian idea of integrating successively over momentum shells. Different choices of $R_{k}$ correspond to different $\mathrm{RG}$ trajectories in the space of all action functionals. But by construction, the complete quantum solution $\Gamma=\Gamma_{k \rightarrow 0}$, being the end point of all trajectories, is independent of $R_{k}$.

This $R_{k}$ independence of the solution, of course, holds only for exact solutions to the flow equation. Approximations such as the choice of a truncation generically introduce a cutoff dependence of the final result. On the one hand, this is clearly a disadvantage of the method; one is led to study one and the same problem with many different cutoffs in order to extract cutoff-independent information. On the other hand, after having accepted that exact solutions might never be at our disposal for most quantum field theories, we can exploit the cutoff dependence in order to improve our approximations.

In order to illustrate this point, let us recall that truncations cut a hypersurface out of the space of all action functionals. A truncation will be acceptable if the complete quantum effective action lies within or close to this hypersurface. But this is not a sufficient criterion: imagine a certain exact RG trajectory (corresponding to a certain cutoff function) that begins and ends within this hypersurface, but in between develops a large distance to the hypersurface. In the exact theory, this flow may largely be driven by operators which do not belong to the truncation spanning the hypersurface. Working only within the truncation, the contribution of these other operators cannot be accounted for, and the so-found solution to the flow will generally be different from the true solution.

Instead, the optimal strategy would be to choose those exact RG trajectories (and their corresponding cutoff functions) that lie completely in (or close to) the hypersurface. But strictly speaking, this ideal case is not possible, since the cutoff function generally couples the flow to all operators, so that an RG trajectory will never lie only within a restricted hypersurface. A more precise criterion would be that the truncated RG trajectory within the hypersurface should be equal to (or close to) the exact RG trajectory after projecting the latter onto the hypersurface. Then, the flow towards the quantum solution is driven mainly by the operators contained in the truncation, and the final result will represent a good approximation to the exact one. However, we are currently not aware of any method that fully formalizes these ideas. Up to now, the properties of the flow that depend on the cutoff function can only be investigated within a given truncation.

However, a systematic study of cutoff functions has recently been put forward mainly within derivative-expansion truncations in scalar and fermionic theories, and "opti- 
mized" cutoff functions have been proposed [17]. The optimization criterion focuses on improving the convergence of approximate solutions to flow equations; in fact, for scalar $O(N)$ symmetric theories, it leads to better results for the critical exponents [18].

Spectrally adjusted cutoff. The class of cutoff functions employed in this work is also considered to be improved in the sense mentioned above. In this case, the improvement does not refer to the precise shape of the cutoff function, but rather to the choice of its argument. Here, we will use not just the spectrum of the Laplace operator (which would be the gauge-covariant generalization of the momentum squared), but the full second functional derivative of the effective average action $\Gamma_{k}^{(2)}$ evaluated at the background field.

The argument of the cutoff function can be understood as a parameter which controls the order and size of the momentum shell that is integrated out upon lowering the scale from $k$ to $k-\Delta k$. It appears natural that a truncated flow can be controlled better if each momentum shell covers an equal part of the spectrum of quantum fluctuations. The spectrum itself is not fixed, but $k$ dependent; lower modes get dressed by integrating out higher modes. In order to adapt the cutoff function to this spectral flow, we insert the full $\Gamma_{k}^{(2)}$ into its argument, and so obtain a "spectrally adjusted" cutoff.

This has two technical consequences: first, as the flow equation is evaluated at the background field in our truncation, the right-hand side can be transformed into a proper time representation; here, we have powerful tools at our disposal that allow us to keep track of the full dependence of the flow equation on the field strength squared. Second, the degree of nonlinearity of the flow equation strongly increases, inhibiting its straightforward analytical or numerical computation even within simple truncations. We solve this technical problem by first expanding the flow for the gauge coupling in an asymptotic series, and then reconstructing an integral representation for this series by analyzing the leading (and subleading) asymptotic growth of the series coefficients. Whereas most parts of our work are formulated in $d$ $>2$ dimensions and for the gauge group $\mathrm{SU}(N)$, this final analysis concentrates on the most interesting cases of $d=4$ and $N=2$ or $N=3$.

Results. As a result, we find a representation of the $\beta$ function of Yang-Mills theory. For weak coupling, we rediscover an accurate perturbative behavior. As the scale $k$ approaches the infrared, the coupling grows and finally tends to an infrared stable fixed point, $\alpha_{\mathrm{s}} \rightarrow \alpha_{*}$. Our quantitative results are

$$
\begin{gathered}
\alpha_{*} \simeq 11.3 \quad \text { for } \mathrm{SU}(2), \\
\alpha_{*} \simeq 7.7 \pm 2 \quad \text { for } \mathrm{SU}(3) .
\end{gathered}
$$

The uncertainty in the SU(3) case arises from an unresolved color structure in our calculation (cf. Appendix E).

The complete flow of the running coupling is depicted in Fig. 1 for pure SU(2) Yang-Mills theory in comparison with perturbation theory. For illustrative purposes, we use $\alpha_{\mathrm{s}}\left(M_{Z}\right) \simeq 0.117$ as the initial value $\left(M_{Z} \simeq 91.2 \mathrm{GeV}\right)$. Sizable deviations from perturbation theory occur for $k$

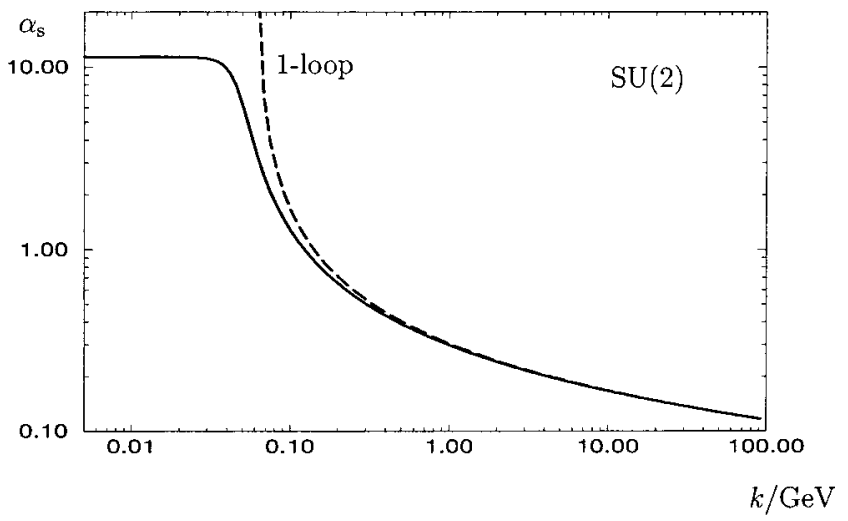

FIG. 1. Running coupling $\alpha_{\mathrm{s}}$ versus momentum scale $k$ in $\mathrm{GeV}$ for gauge group $\mathrm{SU}(2)$, using the initial value $\alpha_{\mathrm{s}}\left(M_{Z}\right) \simeq 0.117$. The solid line represents the result of our calculation in comparison with one-loop perturbation theory (dashed line).

$\$ 1 \mathrm{GeV}$, and the fixed point plateau is reached for $k$ $=\mathcal{O}(10 \mathrm{MeV})$. We shall argue below that a larger truncation as well as the inclusion of dynamical quarks are expected to decrease the value of $\alpha_{*}$.

The paper is organized as follows. Section II briefly recalls the framework of flow equations in gauge theories with the background-field method and describes our basic approximations. In Sec. III we boil down the flow equation as required for our truncation. Section IV is devoted to extracting the RG flow of the running gauge coupling, which is the main result of the present work. The role of the spectrally adjusted cutoff is illustrated in Sec. V. Section VI contains our conclusions and a discussion of our results in the light of related literature.

\section{FLOW EQUATION FOR YANG-MILLS THEORY}

We begin with a brief outline of the flow equation and the background-field formalism as they are employed in this work. We focus on direct applicability and the required approximations and leave aside more formal (though important) aspects, as they are presented in [12] and [19]. Let us therefore start with a more explicit representation of the flow equation for the effective average action,

$$
\begin{aligned}
\partial_{t} \Gamma_{k}[A, \bar{A}]= & \frac{1}{2} \operatorname{STr}\left\{\partial_{t} R_{k}\left(\Gamma_{k}^{(2)}[\bar{A}, \bar{A}]\right)\right. \\
& \left.\times\left[\Gamma_{k}^{(2)}[A, \bar{A}]+R_{k}\left(\Gamma_{k}^{(2)}[\bar{A}, \bar{A}]\right)\right]^{-1}\right\},
\end{aligned}
$$

where we denote the so-called classical gauge field by $A_{\mu}^{a}$, which is the usual field variable of the quantum effective action (conjugate to the source). We also introduce a background field $\bar{A}_{\mu}^{a}$, and have already inserted $\Gamma_{k}^{(2)}$ evaluated at the background field into the cutoff function. ${ }^{1}$ The symbol STr implies tracing over all internal indices and provides for

\footnotetext{
${ }^{1}$ This $\Gamma_{k}^{(2)}$ is evaluated at the background field because an $A$ dependence would spoil the one-to-one correspondence of the flow equation to the functional integral.
} 
a minus sign in the ghost sector. We aim at solving Eq. (4), using the following truncation:

$$
\begin{aligned}
\Gamma_{k}[A, \bar{A}]= & \Gamma_{k}^{\mathrm{inv}}[A]+\Gamma_{k}^{\mathrm{gf}}[A, \bar{A}]+\Gamma_{k}^{\mathrm{gh}}[A, \bar{A}] \\
& +\Gamma_{k}^{\mathrm{gauge}}[A, \bar{A}] .
\end{aligned}
$$

Following [20], the background-field method is introduced to enable us not only to perform a meaningful integration over gauge-fixed quantum fluctuations but to simultaneously arrive at a gauge-invariant effective action. Identifying the quantum fluctuations with $A-\bar{A}$, the gauge-fixing term

$$
\Gamma_{k}^{\mathrm{gf}}[A, \bar{A}]=\frac{1}{2 \alpha} \int_{x}\left\{D_{\mu}[\bar{A}](A-\bar{A})_{\mu}\right\}^{2}
$$

with a gauge parameter $\alpha$ is invariant under a simultaneous gauge transformation of $A_{\mu}^{a}$ and $\bar{A}_{\mu}^{a}$, and so is the ghost action

$$
\Gamma_{k}^{\mathrm{gh}}[A, \bar{A}]=-\int_{x} \bar{c} D_{\mu}[\bar{A}] D_{\mu}[A] c,
$$

where the ghosts $\bar{c}, c$ are understood to transform homogeneously. We should stress that with this truncation of the ghost and gauge-fixing sector, we neglect any running there.

If we solved the theory completely, the resulting quantum effective action $\Gamma_{k=0}[A, \bar{A}]$ would be gauge invariant precisely at $A=\bar{A}$. Imposing a normalization of $\Gamma_{k}^{\text {gauge }}[A, \bar{A}]$ such that

$$
\Gamma_{k}^{\text {gauge }}[A=\bar{A}, \bar{A}]=0
$$

we conclude that the so-found solution $\Gamma_{k \rightarrow 0}^{\text {inv }}[A]$ would be gauge invariant and would represent the desired quantum effective action (provided that we also worked out the complete ghost sector). The quantity $\Gamma_{k}^{\text {gauge }}$ hence parametrizes the gauge-noninvariant remainder of the action in the physically irrelevant case of $A \neq \bar{A}$.

Although we are finally interested in $\Gamma_{k}[A$ $=\bar{A}, \bar{A}], \quad \Gamma_{k}^{\text {gauge }}$ cannot be dropped right from the beginning in Eq. (4), because its second functional derivative $\left(\Gamma_{k}^{\text {gauge }}\right)^{(2)}[A, \bar{A}] \neq 0$ in general; $\Gamma_{k}^{\text {gauge }}$ contributes to the flow of $\Gamma_{k}[A, \bar{A}]$ even at $A=\bar{A}$. Still, neglecting $\Gamma_{k}^{\text {gauge }}$ seems to be a consistent truncation if we are interested only in $\Gamma_{k}^{\text {inv }}[A=\bar{A}]$. But besides Eq. (4), the effective action also has to satisfy the constraints imposed by gauge invariance in the form of the modified Ward identity; in symbolic notation,

$$
\mathcal{L}_{\mathrm{W}}\left[\Gamma_{k}\right]=\Delta\left[R_{k}\right]
$$

where $\mathcal{L}_{\mathrm{W}}$ denotes the usual Ward operator constraining the effective action $\Gamma_{k}$, and the cutoff-dependent right-hand side represents the modification due to the infrared regulator $R_{k}$ (for an explicit representation of Eq. (9), see [12,19]). In fact, if the cutoff is removed in the limit $k \rightarrow 0$, we rediscover the standard Ward identity $\mathcal{L}_{\mathrm{W}}\left[\Gamma_{k}\right]=0$. Inserting our truncation (5) into the Ward identity, the first three terms drop out and we are left with

$$
\mathcal{L}_{\mathrm{W}}\left[\Gamma_{k}^{\text {gauge }}\right]=\Delta\left[R_{k}\right]
$$

This tells us that, on the one hand, $\Gamma_{k}^{\text {inv }}$ is indeed not constrained by the modified Ward identity and any gaugeinvariant ansatz is allowed; on the other hand, a vanishing $\Gamma_{k}^{\text {gauge }}$ is generally inconsistent with the constraint. It is a nontrivial assumption of this work that $\Gamma_{k}^{\text {gauge }}$ as driven by the right-hand side of Eq. (10) does not strongly influence the flow of $\Gamma_{k}^{\text {inv }}$ at $A=\bar{A}$, so that we can safely neglect it in a first approximation.

With regard to our final asymptotic analysis of the running coupling, we can even weaken this assumption a bit: since we reconstruct the $\beta$ function from its asymptotic series expansion by analyzing its leading growth, neglecting $\Gamma_{k}^{\text {gauge }}$ corresponds to assuming that $\Gamma_{k}^{\text {gauge }}$ does not strongly modify this leading growth. In view of the fact that $\Gamma_{k}^{\text {gauge }}$ starts from zero in the ultraviolet and enters the flow only indirectly, this assumption appears rather natural, at least for a large part of the flow.

We should remark that the effective average action $\Gamma_{k}$ has to satisfy another identity that can be derived by considering the response of $\Gamma_{k}$ on gauge transformations of the background field only. This background-field identity is in close relation to the modified Ward identity [19] (for an explicit proof in QED, see [21]), and also imposes a constraint only on $\Gamma_{k}^{\text {gauge }}$ similar to Eq. (10). As has been shown in [19], this identity does not cause further fine-tuning problems which would add to those that are posed by the modified Ward identity.

In summary, solving the flow equation (4) with the truncation (5) will result in an action functional $\Gamma_{k}^{\text {inv }}[A=\bar{A}]$ which is invariant under the background-field transformation. By neglecting $\Gamma_{k}^{\text {gauge }}$, this invariance is not identical to full quantum gauge invariance even at $A=\bar{A}$, since the flow is not completely compatible with the modified Ward identities. This work is based on the assumption that these violations of quantum gauge invariance have little effect on the final result.

\section{EVALUATION OF THE TRUNCATED FLOW}

We shall now solve the flow equation (4) within the truncation (5) (neglecting $\Gamma_{k}^{\text {gauge }}$ ) and with $\Gamma_{k}^{\text {inv }}$ as given in Eq. (2),

$$
\Gamma_{k}^{\mathrm{inv}}[A]=\int_{x} W_{k}(\theta), \quad W_{k}(\theta)=\sum_{i=1}^{\infty} \frac{W_{i}}{i !} \theta^{i}
$$

where $\theta:=\frac{1}{4} F_{\mu \nu}^{a} F_{\mu \nu}^{a}$. An important ingredient of the flow equation is the cutoff function $R_{k}$, which we display as

$$
R_{k}(x)=x r(y), \quad y:=\frac{x}{Z_{k} k^{2}},
$$


with $r(y)$ being a dimensionless function of a dimensionless argument. We include wave-function renormalization constants $Z_{k}$ in the argument of $r(y)$ for reasons to be discussed below. Note that $Z_{k}$ as well as $R_{k}$ itself are matrices in field space; different field variables may be accompanied by different $Z_{k}$ 's and $R_{k}$ 's. The cutoff function $R_{k}$ has to satisfy the following standard constraints:

$$
\begin{gathered}
\lim _{x / k^{2} \rightarrow 0} R_{k}(x)>0, \quad \lim _{k^{2} / x \rightarrow 0} R_{k}(x)=0, \\
\lim _{k \rightarrow \Lambda} R_{k}(x) \rightarrow \infty
\end{gathered}
$$

which guarantee that $R_{k}$ provides for an infrared regularization, ensure that the regulator is removed in the limit $k \rightarrow 0$, and control the ultraviolet limit where $\Gamma_{k \rightarrow \Lambda}=S_{\Lambda}$ should approach its initial condition $S_{\Lambda}$ at the initial ultraviolet scale $\Lambda$. These constraints are met by the representation (12) and translate into constraints for $r(y)$. Since we will identify the argument $x$ with the full $\Gamma_{k}^{(2)}$ at the background field, the first constraint of Eq. (13) must be formulated more strongly,

$$
\lim _{x / k^{2} \rightarrow 0} R_{k}(x)=Z_{k} k^{2}, \quad r(y \rightarrow 0) \rightarrow \frac{1}{y},
$$

in order to guarantee that the one-loop approximation of the flow equation results in the true one-loop effective action. We shall not specify $r(y)$ any further until we employ an exponential cutoff for the final quantitative computation [see Eq. (D4)].

Within the approximations mentioned above, the flow equation (15) can be written as

$$
\begin{aligned}
\partial_{t} \Gamma_{k}[A=\bar{A}, \bar{A}]=\frac{1}{2} \operatorname{STr} \frac{\partial_{t} R_{k}\left(\Gamma_{k}^{(2)}\right)}{\Gamma_{k}^{(2)}+R_{k}\left(\Gamma_{k}^{(2)}\right)} \\
=\frac{1}{2} \operatorname{STr}[(2-\eta) h(y) \\
\left.+\frac{\partial_{t} \Gamma_{k}^{(2)}}{\Gamma_{k}^{(2)}}[g(y)-h(y)]\right]_{y=\Gamma_{k}^{(2)} / Z_{k} k^{2}},
\end{aligned}
$$

where we abbreviated

$$
h(y):=\frac{-y r^{\prime}(y)}{1+r(y)}, \quad g(y):=\frac{r(y)}{1+r(y)} .
$$

In Eq. (15), we also defined the anomalous dimension

$$
\eta:=-\partial_{t} \ln Z_{k}=-\frac{1}{Z_{k}} \partial_{t} Z_{k},
$$

which is matrix valued in field space similarly to $Z_{k}$; different field variables can acquire different anomalous dimensions. We would like to draw attention to the appearance of the term $\sim \partial_{t} \Gamma_{k}^{(2)}$ on the right-hand side of the flow equation. This term arises from writing $\Gamma_{k}^{(2)}$ into the argument of the cutoff function. It reflects the fact that the cutoff adjusts itself under the flow of the spectrum ${ }^{2}$ of $\Gamma_{k}^{(2)}$. Now it is useful to introduce (at least formally) the Laplace transforms $\widetilde{h}(s)$ and $\tilde{g}(s)$ of the functions $h(y)$ and $g(y)$ :

$$
h(y)=\int_{0}^{\infty} d s \tilde{h}(s) \mathrm{e}^{-y s}, \quad g(y)=\int_{0}^{\infty} d s \tilde{g}(s) \mathrm{e}^{-y s} .
$$

These Laplace transforms $\widetilde{h}(s)$ and $\widetilde{g}(s)$ can be viewed as cutoff functions in Laplace space: they should drop off sufficiently fast for large $s$ (small $s$ ) in order to regularize the infrared (ultraviolet). For instance, the infrared constraint (14) translates into

$$
h(0)=\int_{0}^{\infty} d s \tilde{h}(s)=1=\int_{0}^{\infty} d s \tilde{g}(s)=g(0) .
$$

Additional useful identities for these functions are discussed in Appendix D. Furthermore, introducing the functions $\widetilde{H}(s)$ and $\widetilde{G}(s)$ by

$$
\begin{array}{cl}
\frac{d}{d s} \widetilde{H}(s) & =\widetilde{h}(s), \quad \widetilde{H}(0)=0, \\
\frac{d}{d s} \widetilde{G}(s)=\widetilde{g}(s), & \widetilde{G}(0)=0,
\end{array}
$$

a convenient form of the flow equation can be found which reads

$$
\begin{aligned}
\partial_{t} \Gamma_{k}= & \frac{1}{2} \int_{0}^{\infty} \frac{d s}{s}(\widetilde{H}(s)-\widetilde{G}(s)) \partial_{t} \operatorname{STr} \exp \left(-s \frac{\Gamma_{k}^{(2)}}{Z_{k} k^{2}}\right) \\
& +\frac{1}{2} \int_{0}^{\infty} d s \tilde{g}(s) \operatorname{STr}(2-\eta) \exp \left(-s \frac{\Gamma_{k}^{(2)}}{Z_{k} k^{2}}\right) .
\end{aligned}
$$

The great advantage of this form is that the right-hand side of the flow equation has been transformed into a propertime representation. ${ }^{3}$ The (super-)trace calculation reduces to a computation of a heat-kernel trace for which there are powerful techniques available.

\footnotetext{
${ }^{2}$ Although $\Gamma_{k}^{(2)}$ was also used as the argument of the cutoff in [12], the term $\sim \partial_{t} \Gamma_{k}^{(2)}$ has been neglected in that calculation. The necessity of this term was pointed out to us by D. F. Litim.

${ }^{3}$ This representation of the flow equation should not be confused with the so-called proper time RG [22]. The latter represents a RG flow equation that is derived by RG improving one-loop formulas in propertime representation, and has been used in a variety of studies [23]. However, a proper time flow is generally not exact, as was proved in $[24,25]$ : generic proper time flows can neither be mapped onto exact flows in a derivative expansion nor correctly reproduce perturbation theory. By contrast, our flow equation is derived from an exact RG flow equation and corresponds to the generalized proper time flow proposed in [25]. The essential difference to (standard) proper time flows is the inclusion of $\partial_{t} \Gamma_{k}^{(2)}$ terms. In the present work, particularly these terms will be important and will not be neglected. In agreement with [25], our findings therefore suggest that proper time flows may be improved towards exact RG flows by including the $\sim \partial_{t} \Gamma_{k}^{(2)}$ terms systematically.
} 
Within the actual present truncation (neglecting $\Gamma_{k}^{\text {gauge }}$ ),

$$
\Gamma_{k}[A, \bar{A}]=\int_{x} W_{k}(\theta)+\Gamma_{k}^{\mathrm{gh}}[A, \bar{A}]+\Gamma_{k}^{\mathrm{gf}}[A, \bar{A}],
$$

$\Gamma_{k}^{(2)}$ still has a complicated structure which inhibits obtaining general and exact results for the heat-kernel trace. Fortunately, a general solution is not necessary; we merely have to project the right-hand side onto the truncation, implying that we need only the dependence of the right-hand side on the invariant $\theta=\frac{1}{4} F_{\mu \nu}^{a} F_{\mu \nu}^{a}$; other invariants occurring in the heat-kernel trace are of no importance in the present truncation. Now the crucial observation is that the heat-kernel dependence on $\theta$ can be reconstructed by performing the computation for the special field configuration of a covariant constant magnetic field [as it is explicitly defined in Eq. (A1)]. In addition to this, we can perform the computation for $A=\bar{A}$. For this field configuration, the flow equation finally depends only on the field parameter $\theta=\frac{1}{4} F_{\mu \nu}^{a} F_{\mu \nu}^{a}$ $\equiv \frac{1}{2} B^{2}$, where $B$ denotes the strength of the magnetic field; the latter is pseudo-Abelian and points into a single direction in color space, characterized by a color unit vector $n^{a}$. Extracting this $B$ dependence of the heat kernel allows us to reconstruct the flow of $W_{k}\left(\frac{1}{2} B^{2}\right)$.

It should be stressed that considering a covariant constant background field is nothing but a technical trick to project onto the truncation; we do not at all assume that such a background represents the vacuum configuration of YangMills theory, as is the case, e.g., in the Savvidy vacuum model [26].

This trick also allows us to decompose the operator $\Gamma_{k}^{(2)}$ into linearly independent pieces. In particular, the gauge-field fluctuations can be classified into modes with generalized transversal (T) and longitudinal (L) polarization with respect to the magnetic-field direction in spacetime, and into parallel $\|$ and perpendicular $\perp$ modes with respect to the field direction in color space. Introducing the corresponding projectors $P_{\mathrm{L}, \mathrm{T}}$ and $P_{\|, \perp}$ (explicitly defined in Appendix A), the operator $\Gamma_{k}^{(2)}$ can be represented as

$$
\begin{aligned}
\Gamma_{k}^{(2)}= & P_{\mathrm{T}} P_{\perp}\left[W_{k}^{\prime} \mathcal{D}_{\mathrm{T}}\right]+P_{\mathrm{L}} P_{\perp}\left[\frac{1}{\alpha} \mathcal{D}_{\mathrm{T}}\right]+P_{\mathrm{T}} P_{\|}\left[W_{k}^{\prime}\left(-\partial^{2}\right)\right. \\
& \left.+W_{k}^{\prime \prime} \mathrm{S}\right]+P_{\mathrm{L}} P_{\|}\left[\frac{1}{\alpha}\left(-\partial^{2}\right)\right]+P_{\mathrm{gh}}\left[-D^{2}\right],
\end{aligned}
$$

where $\Gamma_{k}^{(2)} \equiv \Gamma_{k}^{(2)}[A=\bar{A}, \bar{A}]$, and we drop the bars from now on. Here we also defined the operators

$$
\begin{aligned}
\left(\mathcal{D}_{\mathrm{T}}\right)_{\mu \nu}^{a b}= & \left(-D^{2} \delta_{\mu \nu}+2 \mathrm{i} \bar{g} F_{\mu \nu}\right)^{a b}, \\
\mathrm{~S}_{\mu \nu} & =\mathrm{F}_{\mu \alpha} \mathrm{F}_{\beta \nu} \partial^{\alpha} \partial^{\beta}, \quad \mathrm{F}_{\mu \nu}=\mathrm{n}^{a} F_{\mu \nu}^{a} .
\end{aligned}
$$

The formal symbol $P_{\mathrm{gh}}$ in Eq. (23) projects onto the ghost sector, and $W_{k}^{\prime} \equiv(d / d \theta) W_{k}(\theta)$; for details about this decomposition, see Appendix A.

At this point, we are free to choose different cutoff wavefunction renormalizations $Z_{k}$ for each of the linearly independent parts in Eq. (23). If we were solving the flow equa- tion exactly, the final result would be independent of this choice; however, for a truncated flow, a clever choice can seriously improve the approximation. With regard to the form of $R_{k}$ in Eq. (12), it is obvious that the $Z_{k}$ 's control the precise position at which the scale $k$ cuts off the infrared of the momentum spectrum. Since the latter is determined by the Laplace-type operators $-\partial^{2},-D^{2}, \mathcal{D}_{\mathrm{T}}$ in Eq. (23), we can cut them off at $k^{2}$ by choosing

$$
Z_{\text {ghost }, k}=1, \quad Z_{\mathrm{L}, k}=\frac{1}{\alpha}, \quad Z_{\mathrm{T}, k}=W_{k}^{\prime}(0) \equiv Z_{\mathrm{F}, k},
$$

for ghost, longitudinal, and transversal fluctuations, respectively. ${ }^{4}$ This choice guarantees that the longitudinal and ghost modes are cut off at the same point, providing for a necessary cancellation. As a side effect, the flow becomes independent of the gauge-fixing parameter $\alpha$, so that we can implicitly choose Landau gauge $\alpha \rightarrow 0$, which is known to be a fixed point of the flow $[7,29]$. Finally, the transversal cutoff wave-function renormalization is set equal to the gauge-field wave function renormalization, which can be read off at the weak-field limit, $\left.\Gamma_{k}[A]\right|_{\text {w.f. }} \simeq W_{k}^{\prime}(0) \theta \equiv\left(Z_{\mathrm{F}, k} / 4\right) F_{\mu \nu}^{a} F_{\mu \nu}^{a}$.

Using trace identities found in [12], the heat-kernel trace occurring in Eq. (21) can be further reduced to

$$
\begin{aligned}
\operatorname{STr} \exp \left(-s \frac{\Gamma_{k}^{(2)}}{Z_{k} k^{2}}\right)= & \operatorname{Tr}_{x \mathrm{~L}} \exp \left[-\frac{s}{k^{2}}\left(\frac{W_{k}^{\prime}}{Z_{\mathrm{F}, k}}\left(-\partial^{2}\right)+\frac{W_{k}^{\prime \prime}}{Z_{\mathrm{F}, k}} \mathrm{~S}\right)\right] \\
& -d \operatorname{Tr}_{x} \exp \left(-\frac{s}{k^{2}} \frac{W_{k}^{\prime}}{Z_{\mathrm{F}, k}}\left(-\partial^{2}\right)\right) \\
& +\operatorname{Tr}_{x \mathrm{cL}} \exp \left(-\frac{s}{k^{2}} \frac{W_{k}^{\prime}}{Z_{\mathrm{F}, k}} \mathcal{D}_{\mathrm{T}}\right) \\
& -\operatorname{Tr}_{x \mathrm{c}} \exp \left(-\frac{s}{k^{2}} \frac{W_{k}^{\prime}}{Z_{\mathrm{F}, k}}\left(-D^{2}\right)\right) \\
& -\operatorname{Tr}_{x \mathrm{c}} \exp \left(-\frac{s}{k^{2}}\left(-D^{2}\right)\right)
\end{aligned}
$$

where the traces can act on spacetime $(x)$, color (c), or Lorentz (L) indices. For the trace in Eq. (23) involving $\eta$ (matrix valued), all terms in Eq. (26) containing $Z_{\mathrm{F}, k}$ will acquire an anomalous-dimension contribution which we will also call $\eta$ for simplicity:

\footnotetext{
${ }^{4}$ In agreement with our approximation of neglecting $\Gamma_{k}^{\text {gauge }}$, we also do not distinguish between the wave-function renormalization constant of the classical field $A$ and that of the background field $\bar{A}$; if $\Gamma_{k}^{\text {gauge }}$ were taken into account, this distinction would have to be made [27]. A similar problem would occur if we relaxed the constraint (14) for the cutoff; by keeping track of different wavefunction renormalization constants, such a problem was explicitly solved in [28].
} 


$$
\eta=-\partial_{t} \ln Z_{\mathrm{F}, k}=-\frac{1}{Z_{\mathrm{F}, k}} \partial_{t} Z_{\mathrm{F}, k} .
$$

The various heat-kernel traces are computed in Appendix B. In order to display the result concisely, let us define the auxiliary functions

$$
f_{1}(u)=\frac{1}{u^{d / 2}}\left(\frac{(d-1)}{2} \frac{u}{\sinh u}+2 u \sinh u\right),
$$

$$
f_{2}(u)=\frac{1}{2} \frac{1}{u^{d / 2}} \frac{u}{\sinh u},
$$

$$
f_{3}\left(v_{1}, v_{2}\right)=\frac{1}{v_{1}^{d / 2}}\left(1-v_{2}\right) .
$$

Equipped with these abbreviations, the flow equation can be written as

$$
\begin{aligned}
\partial_{t} W_{k}(\theta)= & \frac{1}{2(4 \pi)^{d / 2}} \int_{0}^{\infty} d s\left(\tilde { g } ( s ) \left\{\sum_{l=1}^{N^{2}-1}\left[2(2-\eta) f_{1}\left(\frac{s}{k^{2}} \frac{W_{k}^{\prime}}{Z_{\mathrm{F}, k}} \bar{B}_{l}\right)-4 f_{2}\left(\frac{s}{k^{2}} \bar{B}_{l}\right)\right] \bar{B}_{l}^{d / 2}\right.\right. \\
& \left.-(2-\eta) f_{3}\left(\frac{s}{k^{2}} \frac{W_{k}^{\prime}}{Z_{\mathrm{F}, k}}, \frac{W_{k}^{\prime}}{W_{k}^{\prime}+B^{2} W_{k}^{\prime \prime}}\right)\right\} \\
& \left.+\frac{1}{2 s}[\widetilde{H}(s)-\widetilde{G}(s)] \partial_{t}\left[4 \sum_{l=1}^{N^{2}-1}\left(f_{1}-f_{2}\right) \bar{B}_{l}^{d / 2}-2 f_{3}\right]\right),
\end{aligned}
$$

where $\bar{B}_{l}=\bar{g}\left|\nu_{l}\right| B, \quad \bar{g}$ denotes the bare coupling, and $\nu_{l}$ represents the $l=1, \ldots, N^{2}-1$ eigenvalues of the color matrix $\left(n^{a} T^{a}\right)^{b c}$. The auxiliary functions $f_{i}$ in the last line are understood to have the same arguments as in the first lines. It is convenient to express the flow equation in terms of dimensionless renormalized quantities:

$$
\begin{gathered}
g^{2}=k^{d-4} Z_{\mathrm{F}, k}^{-1} \bar{g}^{2}, \\
\vartheta=g^{2} k^{-d} Z_{\mathrm{F}, k} \theta \equiv k^{-4} \bar{g}^{2} \theta, \\
w_{k}(\vartheta)=g^{2} k^{-d} W_{k}(\theta) \equiv k^{-4} Z_{\mathrm{F}, k}^{-1} \bar{g}^{2} W_{k}\left(k^{4} \vartheta / \bar{g}^{2}\right),
\end{gathered}
$$

and evaluate the derivative $\partial_{t}$ from now on at fixed $\vartheta$ instead of fixed $\theta$. As a result, the flow equation (29) turns into

$$
\begin{aligned}
\partial_{t} w_{k}(\vartheta)= & (4-\eta) w_{k}+4 \vartheta \dot{w}_{k}(\vartheta) \\
& +\frac{g^{2}}{2(4 \pi)^{d / 2}}\left\{\int_{0}^{\infty} d s \tilde{h}(s)\left[4 \sum_{l=1}^{N^{2}-1}\left[f_{1}\left(s \dot{w}_{k} b_{l}\right)-f_{2}\left(s b_{l}\right)\right] b_{l}^{d / 2}-2 f_{3}\left(s \dot{w}_{k}, \frac{\dot{w}_{k}}{\dot{w}_{k}+2 \vartheta \ddot{w}_{k}}\right)\right]\right. \\
& -\eta \int_{0}^{\infty} d s \tilde{g}(s)\left[2 \sum_{l=1}^{N^{2}-1} f_{1}\left(s \dot{w}_{k} b_{l}\right) b_{l}^{d / 2}-f_{3}\left(s \dot{w}_{k}, \frac{\dot{w}_{k}}{\dot{w}_{k}+2 \vartheta \ddot{w}_{k}}\right)\right] \\
& +\int_{0}^{\infty} d s[\widetilde{h}(s)-\tilde{g}(s)]\left[\left(\frac{\partial_{t} \dot{w}_{k}}{\dot{w}_{k}}-\frac{4 \vartheta \ddot{w}_{k}}{\dot{w}_{k}}\right) 2 \sum_{l=1}^{N^{2}-1} f_{1}\left(s \dot{w}_{k} b_{l}\right) b_{l}^{d / 2}\right. \\
& \left.\left.-\frac{2}{d}\left(\partial_{t}-4 \vartheta \partial_{\vartheta}\right) f_{3}\left(s \dot{w}_{k}, \frac{\dot{w}_{k}}{\dot{w}_{k}+2 \vartheta \ddot{w}_{k}}\right)\right]\right\}
\end{aligned}
$$

where $\dot{w}_{k}(\vartheta)=\partial_{\vartheta} w_{k}(\vartheta)$, and we abbreviated $b_{l}=\left|\nu_{l}\right| \sqrt{2 \vartheta}$. Equation (31) represents one of the main results of the present work. Within the chosen truncation, this flow equation leads to the full quantum effective action of Yang-Mills theory upon integration from its initial condition at $\Lambda$ down to $k=0$.
As a first comment, we would like to mention that we rediscover the flow equation of [12] if we perform an expansion for weak magnetic field and if we neglect all terms proportional to $\partial_{t} \Gamma_{k}^{(2)}$. In order to isolate the latter from the rest of Eq. (31), the single factor of $\tilde{g}(s)$ in the second line should be represented as $\tilde{h}(s)-[\tilde{h}(s)-\tilde{g}(s)]$, and then all 
terms proportional to $[\widetilde{h}(s)-\tilde{g}(s)]$ should be dropped $[\mathrm{cf}$. Eq. (15)].

Obviously, the $\partial_{t} \Gamma_{k}^{(2)}$ terms $\sim[\widetilde{h}(s)-\tilde{g}(s)]$ modify the flow equation extensively. ${ }^{5}$ They seriously increase the degree of complexity of this partial differential equation, so that neither an analytic nor a numeric evaluation is straightforward. The next section will be devoted to a search for the simplest possible and consistent approximation.

Finally, we remark that the flow equation contains a seeming divergence: in the limit of small $k$, the $s$ integrand may not be bounded for $s \rightarrow \infty$, owing to the last term $\sim \sinh u$ in the auxiliary function $f_{1}$ given in Eq. (28). However, this divergence is well understood and can be controlled. It arises from the Nielsen-Olesen unstable mode [30] in the operator $\mathcal{D}_{\mathrm{T}}$, and can be traced back to the fact that the gluon-spin coupling to the constant magnetic field can lower its energy below zero. Because of this mode, the covariant constant magnetic field is known to be unstable, if considered as the quantum vacuum state of Yang-Mills theory. The divergence can be identified as a pole at complex infinity. The $s$ integral can be properly defined by analytic continuation, resulting in a real part as well as an imaginary part. The real part is indeed important because it contributes to the $\beta$ function and the form of the effective action in a perturbative computation (see below). The imaginary part is interpreted as a measure for the instability of the constant-field vacuum.

As we have stressed before, the constant-magnetic-field background is just a calculational tool in the present context, and the validity of the flow equation is not based on this background. Therefore, the $s$ integral can be properly defined by analytic continuation around this pole at complex infinity. The resulting real part will be a valid and important contribution to the flow, but the imaginary part is of no relevance here. If we were really interested in a constant-field vacuum, the flow generated by this imaginary part would describe how the instability develops upon integrating out the unstable mode in a Wilsonian sense.

\section{RUNNING GAUGE COUPLING IN $\boldsymbol{d}=4$}

In order to find a strategy for solving the flow equation (31) within a first simple approximation, let us take a closer look at the standard procedure employed for ordinary cutoffs without $\partial_{t} \Gamma_{k}^{(2)}$ terms. In such a case, the partial differential equation can be rewritten as an infinite set of coupled ordinary first-order differential equations by expanding the truncation, e.g.,

$$
w_{k}(\vartheta)=\sum_{i=1}^{\infty} \frac{w_{i}}{i !} \vartheta^{i}, \quad w_{1}=1
$$

Note that, owing to the choice (25) for $Z_{k}$ and the definition (30), $w_{1}=1$ is fixed, so that the generalized coupling $W_{1}$ is

\footnotetext{
${ }^{5}$ Incidentally, it is easy to show that no admissible cutoff shape function $r(y)$ exists such that $h(y)=g(y)$. Hence, the $\partial_{t} \Gamma_{k}^{(2)}$ terms are present for all cutoff shape functions.
}

traded for the anomalous dimension $\eta$. As a result, we obtain infinitely many flow equations for the couplings $w_{i}$ which, for an ordinary cutoff, read

$$
\left.\partial_{t} w_{i}\right|_{\text {ordinary cutoff }}=X_{i}\left(\eta, w_{2}, \ldots, w_{i+1}\right), \quad i=2,3, \ldots
$$

Equation (33) is supplemented by an additional equation for $\eta$. The functions $X_{i}$ are obtained as the $i$ th coefficient of the $\vartheta$ series expansion of the flow equation's right-hand side. This infinite tower of equations is then approximated by a finite one by setting all $w_{i}=0$ by hand for some $i>i_{\text {trunc }}$, resulting in $i_{\text {trunc }}$ equations for $i_{\text {trunc }}$ variables. The quality of this further truncation can be checked by varying $i_{\text {trunc }}$.

This recipe cannot be directly applied to the present case involving the spectrally adjusted cutoff because an expansion of the flow equation (31) will be of the form

$$
\begin{aligned}
\partial_{t} w_{i}= & X_{i}\left(\eta, w_{2}, \ldots, w_{i+1}\right) \\
& +Y_{i}\left(\eta, w_{2}, \ldots, w_{i+1} ; \partial_{t} w_{2}, \ldots, \partial_{t} w_{i+1}\right), \\
& i=2,3, \ldots .
\end{aligned}
$$

It is tempting to truncate this tower by setting not only $w_{i>i_{\text {trunc }}}=0$ by hand, but also $\partial_{t} w_{i>i_{\text {trunc }}}=0$. This is too naive, however, because all $\partial_{t} w_{i}$, if understood as the left-hand side of Eq. (34), receive nonzero contributions on the righthand side, even if $i>i_{\text {trunc }}$. Neglecting these right-hand sides would correspond to neglecting some $w_{i}$ 's which are in the truncation $i \leqslant i_{\text {trunc }}$.

In order to apply the above-mentioned recipe, we have to bring Eq. (34) into the form of Eq. (33), i.e., we have to solve for the $\partial_{t} w_{i}$ 's. Formally, this is possible by observing that the functions $Y_{i}$, as they are derived from Eq. (31), are linear in all $\partial_{t} w_{i}$ and $\eta$, and the $X_{i}$ are also linear in $\eta$. Introducing a "vector" $\vec{w}_{t}$ with components

$$
\vec{w}_{t}:=\left\{\begin{array}{l}
w_{t 1}=-\eta \\
w_{t i}=\partial_{t} w_{i} \quad \text { for } \quad i=2,3, \ldots
\end{array}\right\},
$$

Eq. (34) can be written as ${ }^{6}$

$$
w_{t i}=X_{i}\left(w_{2}, \ldots, w_{i+1}\right)+Y_{i j}\left(w_{2}, \ldots, w_{i+1}\right) w_{t j},
$$

or symbolically, $\vec{w}_{t}=\vec{X}+Y \cdot \vec{w}_{t}$. Provided that the operator $1-Y$ is invertible, the desired solution is formally given by

$$
\vec{w}_{t}=\frac{1}{1-Y} \cdot \vec{X}
$$

where the right-hand side is a function of $w_{2}, w_{3}, \ldots$ only. Now, the approximation strategy for the ordinary cutoff can be applied to Eq. (37). Nevertheless, the resulting finite tower of differential equations is substantially different from the ordinary case, even for the smallest $i_{\text {trunc }}$. This is because

\footnotetext{
${ }^{6}$ The meaning of the quantities $X_{i}$ and $Y_{i j}$ changes here slightly, because the $\eta$ and $\partial_{t} w_{i}$ dependence is pulled out compared to Eq. (34).
} 
$X_{i}$ and $Y_{i j}$ are generally nonzero, even for $i, j>i_{\text {trunc }}$, since they depend on the remaining $w_{i \leqslant i}$ trunc (and numbers such as $d$ and $N$ ). And since they are infinite dimensional, we find an infinite number of terms on the right-hand side of the flow equations, in contrast to a finite number for ordinary cutoffs.

For the remainder of this section, we shall evaluate Eq. (37) in the simplest possible way by neglecting all $w_{i}$ 's with $i=2,3, \ldots$ and retaining only the anomalous dimension $\eta$, which is related to the $\beta$ function of Yang-Mills theory via

$$
\beta\left(g^{2}\right) \equiv \partial_{t} g^{2}=(d-4+\eta) g^{2},
$$

so that in $d=4$ we simply have $\beta\left(g^{2}\right)=\eta g^{2}$. We would like to stress that the approximation of neglecting all $w_{i}$ 's at this stage is not at all equal to neglecting them right from the beginning. This further truncation is only consistent after we have disentangled the flows of all $w_{i}$ 's by virtue of Eq. (37).

For the investigation of the $\eta$ equation, corresponding to the first component of the vector equation (37), it is useful to scale out the coupling constant, so that $\vec{X}$ and $Y$ no longer depend on the coupling:

$$
\vec{X} \rightarrow G \vec{X}, \quad Y \rightarrow G Y, \quad G:=\frac{g^{2}}{2(4 \pi)^{d / 2}} .
$$

In $d=4$, the convenient coupling $G$ is related to the standard strong coupling constant $\alpha_{\mathrm{s}} \equiv g^{2} / 4 \pi=8 \pi G$. Using Eq. (39), we can perform a "perturbative" expansion of the $\eta$ equation:

$$
\begin{aligned}
-\eta & \equiv w_{t 1}=\left(\frac{1}{1-G Y}\right)_{1 j} G X_{j} \\
& =G\left(1+G Y+G^{2} Y^{2}+\cdots\right)_{1 j} X_{j}=G\left(\sum_{m=0}^{\infty} G^{m} Y^{m}\right)_{1 j} X_{j} .
\end{aligned}
$$

The explicit representation of $Y$ and $\vec{X}$ can be found by inserting the expansions developed in Appendix C into Eq. (31), and performing the propertime $s$ integration; the latter results in the moments $h_{j}, g_{j}$ of the cutoff functions $\widetilde{h}(s), \tilde{g}(s)$,

$$
h_{j}:=\int_{0}^{\infty} d s s^{j} \widetilde{h}(s), \quad g_{j}:=\int_{0}^{\infty} d s s^{j} \widetilde{g}(s),
$$

which are discussed in Appendix D. In conclusion, we find

$$
\begin{aligned}
X_{i}= & -2^{i+1} \tau_{i} h_{2 i-d / 2} i ! \\
& \times\left((d-2) \frac{\left(2^{2 i}-2\right)}{(2 i) !} B_{2 i}-\frac{4}{(2 i-1) !}\right), \\
Y_{i j}= & A_{i j}+B_{i j}+C_{i j},
\end{aligned}
$$

where $B_{2 i}$ denotes the Bernoulli numbers, and the auxiliary matrices $A, B, C$ are given by $(i, j=1,2, \ldots)$ for matrix $A$

$$
\begin{aligned}
A_{i 1}= & 0 \\
A_{i j}= & 0 \quad \text { if } j>i+1 \\
A_{i j}= & \frac{i !}{(j-1) !}\left[2^{n} \tau_{n}\left(h_{2 n-d / 2}-g_{2 n-d / 2}\right)\right. \\
& \left.\times\left((d-1) \frac{2^{2 n}-2}{(2 n) !} B_{2 n}-\frac{4}{(2 n-1) !}\right)\right]_{n=1+i-j}
\end{aligned}
$$

for matrix B

$$
\begin{aligned}
B_{i j}= & 0 \quad \text { if } \quad j>1 \\
B_{i 1}= & -2^{i} \tau_{i} h_{2 i-d / 2} ! \\
& \times\left((d-1) \frac{2^{2 i}-2}{(2 i) !} B_{2 i}-\frac{4}{(2 i-1) !}\right)
\end{aligned}
$$

and for matrix $\mathrm{C}$

$$
\begin{aligned}
C_{i j} & =0 \quad \text { if } \quad j \neq i+1 \\
C_{i, i+1} & =-\frac{4}{d} i\left(h_{-2}-g_{-2}\right) .
\end{aligned}
$$

These explicit representations (42) and (43c) can be inserted into Eq. (40), and the anomalous dimension and the $\beta$ function can be computed straightforwardly to any finite order in perturbation theory within our truncation.

As an example, let us compute the two-loop $\beta$ function in $d=4$ spacetime dimensions for $\mathrm{SU}(N)$ gauge theories:

$$
\begin{aligned}
\beta\left(g^{2}\right)= & \partial_{t} g^{2} \\
= & -\frac{22 N}{3} \frac{g^{4}}{(4 \pi)^{2}} \\
& -\left(\frac{77 N^{2}}{3}-\frac{127\left(3 N^{2}-2\right)}{45}\right. \\
& \left.\times\left(h_{-2}-g_{-2}\right) h_{2} \tau_{2}\right) \frac{g^{6}}{(4 \pi)^{4}}+\cdots
\end{aligned}
$$

Here we already used the fact that $h_{0}=1=g_{0}$ are independent of the shape of the cutoff function, so that the one-loop coefficient turns out to be universal as it should be and agrees with the standard perturbative result; this should serve as a (rather trivial) check of our computation. Within our truncation, the two-loop coefficient does depend on the cutoff function. In order to compare with [12] where the $\partial_{t} \Gamma_{k}^{(2)}$ terms have been neglected, we choose the exponential cutoff defined in Eq. (D4), implying that $g_{-2}=1, h_{-2}=2 \zeta(3)$ $\simeq 2.404$, and $h_{2}=1 / 6$ as computed in Appendix D. From Appendix E, we take over that $\tau_{2}^{N=2}=2$ and $\tau_{2}^{N=3}=9 / 4$. Inserting all these numbers and comparing this to the perturbative two-loop result, 


$$
\beta_{\text {pert. }}\left(g^{2}\right)=-\frac{22 N}{3} \frac{g^{4}}{(4 \pi)^{2}}-\frac{68 N^{2}}{3} \frac{g^{6}}{(4 \pi)^{4}}+\cdots,
$$

we find a remarkable agreement of $99 \%$ for the two-loop coefficient for SU(2), and $95 \%$ for SU(3). This should be compared to only a $113 \%$ agreement of these coefficients in the case where the $\partial_{t} \Gamma_{k}^{(2)}$ terms are neglected [12]. The inclusion of these terms appears to represent a serious improvement.

However, the picture is not so rosy as it seems to be in view of this result. The reason is that our two-loop result is cutoff-scheme dependent, and we may easily choose a cutoff with a worse agreement at the two-loop level. ${ }^{7}$ Only recently has it been explicitly shown how to obtain the correct scheme-independent two-loop $\beta$ function within the framework of the exact RG [27]; for this, a careful distinction has to be drawn between the running of the coupling with respect to $k$ or the RG scale $\mu$ (see also [31]). Within our truncation here, we can nevertheless turn the argument around by remarking that the exponential cutoff is obviously well suited for the present truncation in the sense that it minimizes the combined effect of the neglected terms such as $w_{2}$, $\left(F_{\mu \nu}^{a} \widetilde{F}_{\mu \nu}^{a}\right)^{2}$, or $\Gamma_{k}^{\text {gauge }}$, etc. on the two-loop coefficient. This particularly justifies the use of the exponential cutoff for an investigation of the complete sum in Eq. (40) and the strongcoupling domain.

Let us summarize what has been achieved so far: in order to extract the flow of the gauge coupling, the flow equation (31) has to be studied near $\vartheta=0$, where the information about $\eta$ is encoded. This suggests an expansion of $w_{k}(\vartheta)$ in powers of $\vartheta$, leading to completely disentangled flow equations (37) for the generalized couplings $\eta, w_{2}, w_{3}, \ldots$. However, since the original flow equation (31) is represented as a parameter integral, its expansion can be asymptotic, which implies that the series expansion in terms of the coupling $G$ in Eq. (40) will be asymptotic as well. This agrees with the general expectation that perturbative expansions of quantum field theories are generically asymptotic.

In practice, this means that the coefficients (for later convenience, we shift the index $m$ here)

$$
a_{m}:=-\left(Y^{m-1}\right)_{1 j} X_{j}, \quad m=1,2, \ldots
$$

in Eq. (40) grow rapidly, so that any arbitrarily large but finite truncation of the series does not make sense. It turns out that these coefficients grow even more strongly than factorially and alternate in sign for the exponential cutoff (we shall comment on other cutoffs later). This does not mean that any physical meaning is lost, but, loosely speaking, that

\footnotetext{
${ }^{7}$ Actually, this point is even more subtle [27]: cutoff-scheme independence of the two-loop $\beta$ function coefficient holds only for mass-independent regulators. The regulator $R_{k}$ obviously does not belong to this class, so that cutoff-scheme dependence has to be expected. However, since we have no information about the true two-loop coefficient for our $k$-dependent regulator $R_{k}$, we shall still use the two-loop coefficient for a mass-independent scheme as our benchmark test.
}

we have expanded an integrand which we should not have expanded. Yet, there are well-defined mathematical tools for reconstructing the integrand representation out of the diverging sum [32]. In other words, we are looking for a (welldefined) integral representation that upon asymptotic expansion leads to a series that agrees with Eq. (40). As is known also from various physical examples [33], just taking only the leading growth of the coefficients into account leads to a good approximation of the integral representation.

Concerning the coefficients $a_{m}$, the leading growth (LG) can be isolated in the term that contains the highest component of $\vec{X}$, i.e., $X_{m}$, yielding

$$
\begin{aligned}
& a_{1}^{\mathrm{LG}}=-X_{1}, \quad a_{2}^{\mathrm{LG}}=-Y_{12} X_{2}, \\
& a_{m}^{\mathrm{LG}}=-Y_{12} Y_{23} Y_{34} \ldots Y_{m-1, m} X_{m} .
\end{aligned}
$$

Inserting the representations (43c) into Eq. (47) for the exponential cutoff, we find

$$
\begin{aligned}
a_{m}^{\mathrm{LG}}= & 4(-2 c)^{m-1} \frac{\Gamma\left(m+3\left(N^{2}-1\right)\right) \Gamma(m+1)}{\Gamma\left(3 N^{2}-2\right)} \tau_{m} \\
& \times B_{2 m-2}\left(2 \frac{2^{2 m}-2}{(2 m) !} B_{2 m}-\frac{4}{\Gamma(2 m)}\right),
\end{aligned}
$$

where we abbreviated $c=2 \zeta(3)-1$. Let us first concentrate on $\mathrm{SU}(2)$, where $\tau_{m}=2$ for $m=1,2, \ldots$ (see Appendix E); let us nevertheless retain the $N$ dependence in all other terms in order to facilitate the generalization to $\mathrm{SU}(3)$.

Actually, Eq. (48) also contains subleading terms. First, we observe that the last term $\sim 1 / \Gamma(2 \mathrm{~m})$ is negligible compared to the term $\sim B_{2 m}$ for large $m$. Nevertheless we also retain this subleading term, since the $m=1$ term contributes significantly to the one-loop $\beta$-function coefficient which we want to maintain in our approximation. Furthermore using the identity

$$
B_{2 m-2}=\frac{(-1)^{m} \Gamma(2 m-1)}{2^{2 m-3} \pi^{2 m-2}} \zeta(2 m-2),
$$

it is tempting to use the $\zeta$-function representation

$$
\zeta(z)=\sum_{l=1}^{\infty} \frac{1}{l^{z}}
$$

and retain only the $l=1$ term, since the others are subleading. Whereas this approximation is indeed justified for the Bernoulli number $B_{2 m}$ in Eq. (48), we have to retain the full $\zeta$ function for the $B_{2 m-2}$ factor, since here we encounter the $\zeta$ function at zero argument for $m=1$ where Eq. (50) is no longer valid. In conclusion, we resum the complete coefficient $a_{m}^{\mathrm{LG}}$ as displayed in Eq. (48), including the leading and also subleading terms.

In the spirit of Borel summation [32], we introduce integral representations for the special functions occurring in Eq. (48), in particular the representation [34] 


$$
\begin{aligned}
\Gamma(2 m-1) \zeta(2 m-2) & =\frac{1}{1-2^{3-2 m}} \int_{0}^{\infty} d t \frac{\mathrm{e}^{t}}{\left(e^{t}+1\right)^{2}} t^{2 m-2}, \\
m & >1 / 2
\end{aligned}
$$

for the $\zeta$ function in Eq. (49), and an Euler $B$ function representation for a combination involving the last term in Eq. (48):

$$
\begin{aligned}
\frac{\Gamma\left(m+3\left(N^{2}-1\right)\right) \Gamma(m+1)}{\Gamma(2 m)} \\
\equiv \frac{\Gamma\left(m+3\left(N^{2}-1\right)\right)}{\Gamma(m)} \frac{\Gamma(m+1)}{\Gamma(m)} B(m, m) \\
=m(m+1) \ldots\left(m+3 N^{2}-4\right) \cdot m \\
\quad \times \int_{0}^{1} d s s^{m-1}(1-s)^{m-1}
\end{aligned}
$$

$$
=\int_{0}^{1} d s\left[\left(\frac{d}{d s}\right)^{\left(3 N^{2}-3\right)} s^{m+\left(3 N^{2}-4\right)}\right]\left(\frac{d}{d s^{\prime}} s^{\prime m}\right)_{s^{\prime}=1-s}
$$

For the remaining $\Gamma$ functions, we use the standard Euler representation. Exploiting these identities, we are able to resum Eq. (40) to this order:

$$
\eta=-G\left(\sum_{m=1}^{\infty} G^{m-1} Y^{m-1}\right)_{1 j} X_{j}^{\mathrm{LG}} \simeq \sum_{m=1}^{\infty} a_{m}^{\mathrm{LG}} G^{m}=: \eta_{\mathrm{a}}+\eta_{\mathrm{b}}
$$

where $\eta_{\mathrm{a}}$ is related to the term $\sim B_{2 m}$ in Eq. (48), whereas $\eta_{\mathrm{b}}$ is related to the term $\sim 1 / \Gamma(2 m)$. The integral representation of $\eta_{\mathrm{a}}$ reads

$$
\eta_{\mathrm{a}}^{N=2}=\frac{32 N G}{\Gamma\left(3 N^{2}-2\right) \pi^{2}} \sum_{l=1}^{\infty} \int_{0}^{\infty} d s_{1} d s_{2} d t \frac{\mathrm{e}^{t-\left(s_{1}+s_{2}\right)}}{\left(\mathrm{e}^{t}+1\right)^{2}} \frac{s_{1} s_{2}^{3 N^{2}-3}}{l^{2}}\left[S\left(\frac{c G s_{1} s_{2} t^{2}}{2 \pi^{4} l^{2}}\right)-\frac{1}{2} S\left(\frac{c G s_{1} s_{2} t^{2}}{8 \pi^{4} l^{2}}\right)\right],
$$

where we defined the sum

$$
-\sum_{m=1}^{\infty} \frac{(-q)^{m-1}}{1-2^{3-2 m}}=1+\sum_{j=0}^{\infty} \frac{q}{2^{j}+\frac{q}{2^{j}}}=: S(q) .
$$

The first sum arises from the asymptotic expansion and is strictly valid only for $|q|<1$; however, the second sum is valid for arbitrary $q$, apart from simple poles at $q=-2^{2 j}$, and rapidly converging, so that this equation should be read from right to left.

The second part $\eta_{\mathrm{b}}$ deserves a comment: as it arises from the last term in Eq. (48), $\sim 1 / \Gamma(2 \mathrm{~m})$, it originates in the last term $\sinh u$ of the auxiliary function $f_{1}$ in Eq. (28), which stems from the lower end of the spectrum; in particular, it contains the Nielsen-Olesen unstable mode. This mode is reflected in a simple pole in the following integral representation for $\eta_{\mathrm{b}}$. This pole gives rise to an imaginary part of the full integrand. As we have stressed above, the imaginary part created by this unstable mode is of no relevance for the flow equation here, so that the proper treatment of the integral results in a principal-value prescription maintaining the important real part. For a numerical realization, this prescription can best be established by rotating the $t$ integral arising from Eq. (51) by an angle of, e.g., $\pi / 4$ from the real axis into the upper complex plane and then taking the real part. In conclusion, we get

$$
\eta_{\mathrm{b}}^{N=2}=-\left.\frac{32 N G}{\Gamma\left(3 N^{2}-2\right)} \operatorname{Re} \int_{0}^{1} d s \int_{0}^{\infty} \frac{(1+\mathrm{i})}{\sqrt{2}} d t \frac{\mathrm{e}^{[(1+\mathrm{i}) / \sqrt{2} t]}}{\left(\mathrm{e}^{[(1+\mathrm{i}) / \sqrt{2} t]}+1\right)^{2}}\left(\frac{d}{d s}\right)^{\left(3 N^{2}-3\right)} \frac{d}{d s^{\prime}} s^{3 N^{2}-3} s^{\prime} S\left(-\mathrm{i} \frac{c G s s^{\prime} t^{2}}{2 \pi^{2}}\right)\right|_{s^{\prime}=s-1} .
$$

Although it seems that we have seriously complicated the problem by trading the single $m$ sum in Eq. (40) for a number of integrals and sums, we stress that all integrals and sums in Eqs. (54) and (56) are finite and well defined.

Before we present numerical evaluations of these integrals and sums, let us discuss some features analytically. For small coupling $G=g^{2} /\left[2(4 \pi)^{2}\right]$, we can again expand the integrals asymptotically and obtain

$$
\eta_{\mathrm{a}}=\frac{2}{3} N \frac{g^{2}}{(4 \pi)^{2}}+\cdots, \quad \eta_{\mathrm{b}}=-8 N \frac{g^{2}}{(4 \pi)^{2}}+\cdots,
$$

so that we rediscover the one-loop $\beta$ function [cf. Eq. (44)] as a check. Next, we observe that $\eta_{\mathrm{a}}$ (containing the true leading-order growth of the $a_{m}^{\mathrm{LG}}$, s) is positive not only for 


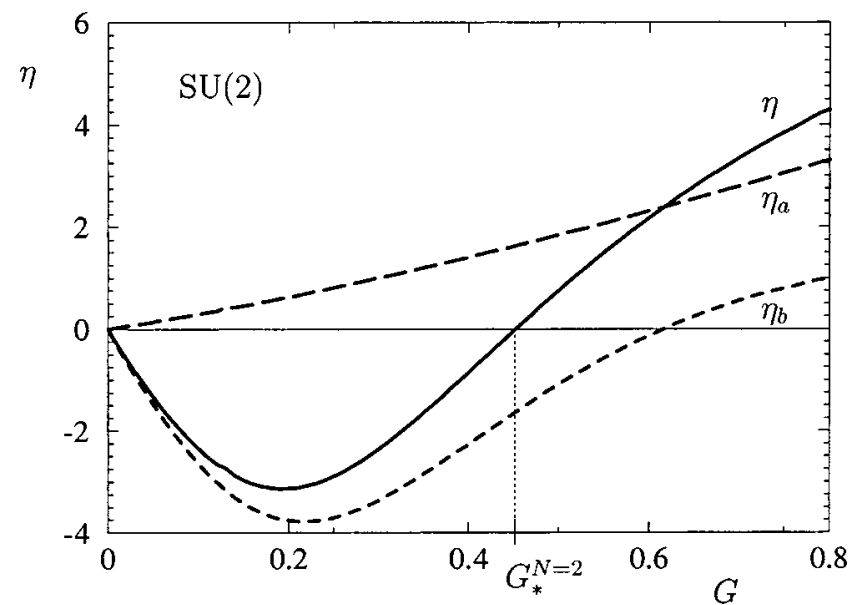

FIG. 2. Anomalous dimension $\eta=\beta\left(g^{2}\right) / g^{2}$ for SU(2) YangMills theory in $d=4$ versus $G=g^{2} /\left[2(4 \pi)^{2}\right]$. The long-dashed line represents the contribution $\eta_{a}$, the short-dashed line $\eta_{b}$, as defined in Eqs. (54) and (56); the solid line is the sum of both.

small but arbitrary $G$. In order to extract large- $G$ information, we note that the sum $S(q)$ can be fitted by

$$
S(q) \simeq c_{1} \sqrt{\frac{1}{c_{1}^{2}}-c_{2} \sqrt{q}+q}, \quad c_{1} \simeq 2.27, \quad c_{2} \simeq 0.7
$$

within $1 \%$ accuracy, implying that $S(q) \simeq c_{1} \sqrt{q}$ for large $q$. In this limit, which corresponds to large $G, \quad \eta_{\text {a }}$ can be evaluated analytically and we find

$$
\begin{aligned}
\eta_{\mathrm{a}}^{N=2}(G \gg 1) & \simeq \frac{24 N c_{1}}{\pi^{4}} \sqrt{\frac{c}{2}} \zeta(3) \ln 2 \Gamma(5 / 2) \\
& \times \frac{\Gamma\left(3 N^{2}-3 / 2\right)}{\Gamma\left(3 N^{2}-2\right)} G^{3 / 2} \simeq 3.24 G^{3 / 2} .
\end{aligned}
$$

Without going into details, we note that there exist fits for $S(-\mathrm{i} q)$ similarly to Eq. (58) involving a square-root behavior for large $q$ (large $G$ ). It turns out that the $G^{3 / 2}$ coefficient vanishes exactly, so that ${ }^{8}$

$$
\eta_{\mathrm{b}}^{N=2} \sim+G^{1 / 2}
$$

Obviously, $\eta_{\mathrm{b}}$ is subleading for large $G$, which agrees with the fact that it arises from subleading parts in the coefficients $a_{m}^{\mathrm{LG}}$. Moreover, $\eta_{\mathrm{b}}$ becomes positive for large $G$, so that there should be a zero in between. This is already the first sign of an infrared stable fixed point at which $\eta\left(G_{*}\right)=0$.

For a numerical evaluation of $\eta_{\mathrm{a}}$ and $\eta_{\mathrm{b}}$, we employ the representations given in Appendix F. We depict the anoma-

\footnotetext{
${ }^{8} \mathrm{We}$ do not evaluate the precise coefficient of the $G^{1 / 2}$ here, since the large- $G$ expansion introduces artificial singularities for the $t$ integration at $t \rightarrow 0$. A more careful treatment reveals that the coefficient is positive.
}

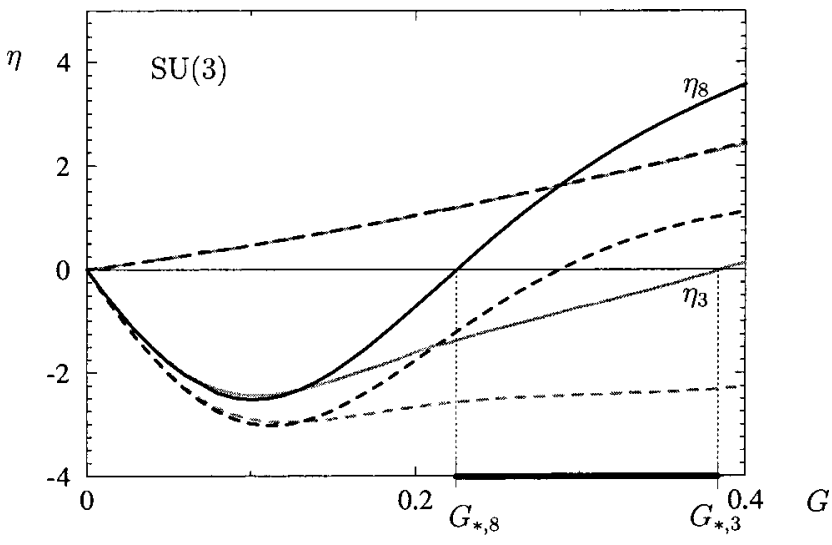

FIG. 3. Anomalous dimension $\eta=\beta\left(g^{2}\right) / g^{2}$ for SU(3) YangMills theory in $d=4$ versus $G=g^{2} /\left[2(4 \pi)^{2}\right]$. The black lines correspond to $\eta_{8}^{N=3}$, the grey lines to $\eta_{3}^{N=3}$, as defined in Eq. (62). The meaning of the dashed lines is as in Fig. 2.

lous dimension $\eta$ and its parts $\eta_{\mathrm{a}}$ and $\eta_{\mathrm{b}}$ in Fig. 2 for the gauge group $\mathrm{SU}(2)$. The plots agree with the analytical estimates given above, and we find an infrared stable fixed point at

$$
G_{*}^{N=2} \simeq 0.45 \Rightarrow \alpha_{*}^{N=2} \simeq 11.3 .
$$

By virtue of Eq. (38), the running gauge coupling approaches this fixed point upon lowering the scale $k$ in the infrared, implying scale invariance. The complete flow of the coupling is obtained by integrating $\beta\left(g^{2}\right) \equiv \partial_{t} g^{2}=\eta g^{2}$ and has been plotted already in Fig. 1.

For the gauge group SU(3), we do not have the explicit representation of the color factors $\tau_{m}$ at our disposal. As discussed in Appendix E, we instead study the two extremal cases for the color vector $n^{a}$ pointing into the 3 or 8 direction in color space. Inserting the corresponding quantities $\tau_{i, 3}^{N=3}$ or $\tau_{i, 8}^{N=3}$ as found in Eq. (E5) into Eq. (48) allows us to display the anomalous dimension $\eta^{N=3}$ in terms of the formulas deduced for $\mathrm{SU}(2)$ :

$$
\begin{aligned}
& \eta_{3}^{N=3}=\left.\frac{2}{3} \eta^{N=2}\right|_{N \rightarrow 3}+\left.\frac{1}{3} \eta^{N=2}\right|_{N \rightarrow 3, c \rightarrow c / 4}, \\
& \eta_{8}^{N=3}=\left.\eta^{N=2}\right|_{N \rightarrow 3, c \rightarrow 3 c / 4} .
\end{aligned}
$$

The notation here indicates that the quantities $N$ and $c$ $=2 \zeta(3)-1$ appearing on the right-hand sides of Eqs. (54) and (56) will be replaced in the prescribed way. Figure 3 depicts our numerical results, and we identify the position of the infrared fixed point in the interval

$$
\begin{aligned}
G_{*}^{N=3} & =\left[G_{*, 8}, G_{*, 3}\right] \\
& \simeq[0.225,0.385] \Rightarrow \alpha_{*}^{N=3} \simeq[5.7,9.7] .
\end{aligned}
$$

This uncertainty of the precise position of the fixed point is not a shortcoming of the techniques involved (e.g., using the covariant-constant magnetic background), but is due to our ignorance of the exact color factors $\tau_{m}$. 
Let us conclude this section with some remarks on the resummation: firstly, we should stress that the results for the fixed point are derived from a resummation of leading and subleading parts of the complete asymptotic series (40). We have checked that the sub-subleading parts (not included in the present resummation) alternate in sign, so that their contribution will be regular. However, we were not able to systematize the sub-subleading terms in a way that they can be resummed further in a consistent way.

Secondly, we performed the computation for the exponential cutoff. It would be desirable to test the stability of the fixed point by using different cutoffs. Unfortunately, we could not find another cutoff shape function $r(y)$ for which the resummation could be done. For many cutoff shape functions used in the literature, the series is also asymptotic and alternating, but the sign changes not from one coefficient to the other but from one group of coefficients to the next, i.e., $a_{n_{1}} \ldots a_{n_{2}}>0$ and $a_{n_{2}+1} \ldots a_{n_{3}}<0$ for $n_{1}<n_{2}<n_{3}$, etc. The outstanding role of the exponential cutoff may be attributed to its close relation to the Bernoulli numbers and their properties.

Let us finally stress once more that the generalized Borel resummation of Eq. (40) does not represent an uncontrolled extrapolation of finite-order perturbation theory. As we have the exact all-order result at our disposal, the resummation corresponds simply to an-also mathematically-welldefined transformation of a series into an integral.

\section{THE ROLE OF THE SPECTRALLY ADJUSTED CUTOFF}

This short section is devoted to a heuristic discussion of the special role played by the spectrally adjusted cutoff in this work, focusing on the truncation employed. The spectral adjustment of the cutoff function to the spectral flow of $\Gamma_{k}^{(2)}$ arises from two sources: first, from using $\Gamma_{k}^{(2)}$ in the argument of $R_{k}$ and, second, from including a carefully chosen wave-function renormalization constant $Z_{k}$ in the cutoff. The latter technique is well known in the problem of calculating anomalous dimensions in scalar and fermionic theories. In order to get a feeling for these two improvements, let us first consider the flow equation, neglecting all $\partial_{t} \Gamma_{k}^{(2)}$ and $\partial_{t} Z_{k}$ terms. For the anomalous dimension, we would then obtain

$$
\eta=-b_{0} \frac{g^{2}}{(4 \pi)^{2}}-b_{1} \frac{g^{2}}{(4 \pi)^{2}} w_{2},
$$

with some coefficient $b_{1}$, and $b_{0}$ being the correct one-loop result. Obviously, choosing furthermore the truncation $w_{2}, w_{3}, \ldots=0$ leaves us with a purely perturbative lowestorder result. This means that all nonperturbative information is contained in the flow of $w_{2}$, which in turn can be reliably computed only by including $w_{3}$, etc. A good estimate therefore probably requires a very large truncation. Even if the precise infrared values of the higher couplings $w_{i}$ may not be very important, their flow exerts a strong influence on the running coupling in this approximation.
Let us now take the $\partial_{t} Z_{k}$ terms into account, but still neglect the $\partial_{t} \Gamma_{k}^{(2)}$ terms. In this case, the flow equation results in the following expression for the anomalous dimension:

$$
\eta=-\frac{b_{0} \frac{g^{2}}{(4 \pi)^{2}}+b_{1} \frac{g^{2}}{(4 \pi)^{2}} w_{2}}{1+d_{1} \frac{g^{2}}{(4 \pi)^{2}}+d_{2} \frac{g^{2}}{(4 \pi)^{2}} w_{2}},
$$

with further coefficients $d_{1}, d_{2}$, where $d_{1}<0$. Particularly this $d_{1}$ makes an important contribution to the two-loop $\beta$ function coefficient. Contrary to Eq. (64), this equation contains information to all orders in $g^{2}$, even for the strict truncation $w_{2}, w_{3}, \ldots=0$. We have to conclude that an adjustment of the cutoff function using a cutoff wave-function renormalization $Z_{k}$ is an effective way to put essential information of the flow of the higher couplings $w_{2}, w_{3}, \ldots$ into $\eta$. In other words, the truncated RG trajectory better exploits the degrees of freedom left in the truncation. Let us note in passing that the flow governed by Eq. (65) runs into a kind of Landau pole for $g^{2} /(4 \pi)^{2} \simeq 1 /\left|d_{1}\right|$, even if the flows of $w_{2}$ and higher couplings are included. This "disease" has occurred in many flow equation studies in Yang-Mills theory $[5,7,12,35]$.

Now let us turn to the full flow equation, including the terms generated by $\partial_{t} \Gamma_{k}^{(2)}$. As explained in the preceding section, the right-hand side cannot be displayed in terms of the $w_{i}$ 's in closed form, because infinitely many terms contribute. Even if we set all $w_{i}$ 's to zero, which indeed corresponds to our final approximation, the anomalous dimension reads

$$
\eta=-\frac{b_{0} \frac{g^{2}}{(4 \pi)^{2}}+b_{1} \frac{g^{4}}{(4 \pi)^{4}}+b_{2} \frac{g^{6}}{(4 \pi)^{6}}+\cdots}{1+d_{1} \frac{g^{2}}{(4 \pi)^{2}}+d_{2} \frac{g^{4}}{(4 \pi)^{4}}+d_{3} \frac{g^{6}}{(4 \pi)^{6}}+\cdots},
$$

with some real coefficients $b_{i}$ and $d_{i}$ [expanding Eq. (66) in powers of $g^{2}$ results in Eq. (40)]. Whereas the nonperturbative dependence of $\eta$ in Eq. (65) resembles that of a Dyson series and is controlled by one coefficient ( $d_{1}$ in that case), Eq. (66) contains nonperturbative information from infinitely many coefficients. The latter arises from the flows $\partial_{t} w_{i}$ which all contribute to Eq. (66). We conclude that the spectrally adjusted cutoff provides for an efficient reorganization of the flow equation, so that a small truncation can contain information which, for ordinary cutoffs, is distributed over infinitely many couplings of a larger truncation. From this observation, we conjecture that the spectrally adjusted cutoff selects a truncated RG trajectory which is "optimized" with respect to the degrees of freedom within a chosen truncation. We furthermore conjecture that this trajectory does not flow into regions of theory space where the exact flow would be mainly driven by couplings which are not contained in the truncation, but is always driven by the couplings within the 
truncation in an optimized way. Whether the truncated RG trajectory flows to the true quantum action or not depends, of course, on the quality of the truncation.

We have obviously verified these conjectures only for a truncation $(\eta)$ within a truncation $\left(\eta, w_{i}\right)$. In fact, in order to exploit the properties of the spectrally adjusted cutoff, we first have to discuss the flow of a larger truncation, then disentangle the flows of the single couplings and finally restrict the calculation to the most relevant part under consideration.

Let us finally point out that using the spectrally adjusted cutoff necessarily requires introducing a background field, because $\Gamma_{k}^{(2)}$ in the cutoff function is not allowed to depend on the actual field variable. A background field generally complicates the formulation, and the technical advantages of the spectrally adjusted cutoff may be compensated for by these further complications. Gauge theories, however, may serve as a natural testing ground for the spectrally adjusted cutoff, since the background-field formalism is advantageous here for further reasons.

\section{CONCLUSIONS}

Starting from the exact renormalization group flow equation for the effective average action in SU(N) Yang-Mills theory in $d$ dimensions, we derive within a series of systematic approximations the $\beta$ function of the gauge coupling. In $d=4$ spacetime dimensions, the resulting flow of the gauge coupling exhibits accurate perturbative behavior and approaches a fixed point in the infrared. The fixed-point results are displayed in Eqs. (61) and (63) for gauge groups SU(2) and $\mathrm{SU}(3)$.

In view of the approximations involved, a number of improvements are desirable in order to confirm the existence of the infrared fixed point. Above all other possible improvements, such as enlarged truncations and explicit cutoff-shape independence (or insensitivity), a better control of gauge invariance under the flow is necessary.

Nevertheless, in view of the flow equation studies performed in the literature for gauge theories so far, it is already remarkable that our approximation to the exact flow equation is integrable down to $k \rightarrow 0$; in many instances, the truncation revealed an explicit insufficiency by developing a Landaupole type of singularity at some finite $k$ in one or more couplings. The new technique in the present work is the use of a cutoff function that adjusts itself permanently to the actual spectrum under the flow. From a practical viewpoint, this cutoff condenses information, which is usually distributed over the flow equations of infinitely many couplings, into the flow equation of a single coupling (in this case the gauge coupling). We have reason to believe that the information, which is reorganized in this way into a single flow equation, is the relevant information that mainly drives the flow of the corresponding coupling. The fact that we improved the agreement with the perturbative two-loop running from merely $113 \%$ to $99 \%$ for $\mathrm{SU}(2)$ (using the exponential cutoff shape function) may serve as a hint in this direction.

If the fixed point exists and our truncation even covers the true mechanism, it is still unlikely that our present results for $\alpha_{*}$ are also quantitatively correct. We expect a lowering of $\alpha_{*}$ for larger truncations owing to the following argument: in our calculation, the position of $\alpha_{*}$ is strongly governed by those modes which are also responsible for asymptotic freedom (contained in $\eta_{\mathrm{b}}$ ). If, in a larger truncation, operators of higher order are generated under the flow, these modes will generically lose influence, and the effects of the remaining spectrum contributing to $\eta_{\mathrm{a}}$ will be enhanced. This will shift $\alpha_{*}$ to smaller values.

A similar effect occurs upon the inclusion of quark degrees of freedom. The perturbative quark contribution to the $\beta$ function is already positive. And since no ultraviolet stable fixed point is known in QED, we also do not expect negative quark contributions beyond the perturbative regime. Therefore, we expect not only the presence of the fixed point in full QCD, but also a substantial shift towards lower values of $\alpha_{*}$. Work in this direction is in progress.

A comparison of our result with the literature is in order now, although it is generally difficult, owing to the various nonperturbative definitions of the gauge coupling; different definitions may agree perturbatively, but differ beyond perturbation theory. Our definition is standard in pure continuum gauge theory; moreover, it is equal to the interaction strength of static quarks with the gauge field. Nevertheless, it is not immediately clear to us how it can be related to a definition which is used, for instance, in lattice gauge theory [36]. This may serve as a word of caution.

The notion of an infrared fixed point for the gauge coupling has been used extensively in recent years, especially in connection with the phenomenology of power corrections in QCD [37]. Furthermore, such a so-called freezing of the coupling has been discussed in phenomenological low-energy models [38], and deduced from an analysis of the famous $R_{e^{+} e^{-}}$ratio [39].

There are also various theoretical arguments favoring an infrared fixed point, e.g., even within a perturbative framework for a finite number of flavors [40]. Furthermore, investigating analyticity properties in the time-like and space-like (Euclidean) region, a scheme called analytic perturbation theory has been proposed, yielding an infrared finite coupling [41]; this program has been successfully applied to hadron and lepton-hadron phenomenology [42]. Having the above-mentioned reservations in mind concerning the various different nonperturbative definitions of the coupling, the question of how they are related to each other deserves further study.

Moreover, an actual nonperturbative computation of gluon and ghost propagators has been set up in the framework of truncated Schwinger-Dyson equations in Landau gauge [43], revealing an infrared fixed point; these results also receive some support from lattice calculations [44]. Again, the relation to our results is not immediately obvious, since the running coupling as defined in [43] is obtained from the ghost-gluon vertex; furthermore, a nonperturbative treatment of the ghost sector turned out to be crucial in that work, but the four-gluon vertex was neglected. Nevertheless, there are also similarities: on very general grounds, it was found in the approximation of [43] that the fixed point scales with the number of colors as $\alpha_{*} \sim 1 / N$. We observe that the 
central value of our $\mathrm{SU}(3)$ result and the $\mathrm{SU}(2)$ result fulfill exactly this relation, although this is far from self-evident in our calculation.

Let us finally discuss further implications of our result: comparing the full $\beta$ function with its perturbative counterpart, we observe a quantitative agreement up to $\alpha_{\mathrm{s}} \sim 1$. This does not, of course, justify the use of perturbation theory up to $\alpha_{\mathrm{s}} \sim 1$ in general, but may explain why perturbation theory gives an accurate answer to some questions, even at its validity limit.

Concerning the low-energy fixed-point region, one may ask whether our result provides for some signals of confinement and an expected mass gap in gauge theories. In the first place, the answer is no, since a strong coupling does not necessarily imply confinement. It is rather likely that the strong coupling of the gauge fields is necessary to give rise to a change of the effective degrees of freedom. These degrees of freedom (not necessarily included in our truncation) with probably nontrivial topological properties will then act as "confiners." Also the picture of confinement arising in the framework of Landau-gauge Dyson-Schwinger equations [43] cannot be contained in our truncation, since it is based on an infrared enhancement of the ghosts which are treated rather poorly in the present work. Improvements in this direction are also subject to future work. As far as a mass gap is concerned, the infrared fixed point behavior is compatible with such a gap; this is because a mass gap cuts off all quantum fluctuations of lower momentum, so that nothing remains to drive the flow. But the mere existence of an infrared fixed point does not require a mass gap.

An indirect signal of a mass gap may be found in the analysis of the different spectral contributions; as we have mentioned above, the perturbative $\beta$ function is mainly determined by the lowest modes in the spectrum, i.e., the lowest Landau levels in the covariant-constant field analysis. As is familiar from QED calculations, the lowest-Landau-level approximation is always appropriate if the field strength exceeds the mass of the fluctuating particle. This is certainly the case in the perturbative domain where the gluon is massless; hence the picture is complete. When we enter the infrared fixed-point region, the contributions from the remaining part of the spectrum $\eta_{\mathrm{a}}$ become important. In the Landaulevel picture, this is always the case if a mass of the order of the lowest Landau level and beyond is present. The value of the mass then controls the influence of the remaining spectrum. Therefore, the influence of the complete spectrum at the fixed point may be a hint for a hidden new mass scale in low-energy Yang-Mills theory.

\section{ACKNOWLEDGMENTS}

The author would like to thank D. F. Litim and J. M. Pawlowski for numerous discussions, for comments on the manuscript, and for communicating their results of Refs. [24] and [25] prior to publication. The author is also grateful to R. Alkofer, W. Dittrich, G.V. Dunne, C.S. Fischer, K. Langfeld, J.I. Latorre, S. Sint and C. Wetterich for helpful information and correspondence, and he wishes to thank W. Dittrich for carefully reading the manuscript. This work is supported by the Deutsche Forschungsgemeinschaft under contract Gi 328/1-1.

\section{APPENDIX A: DECOMPOSITION OF $\Gamma_{k}^{(2)}$}

Here we briefly describe the method developed in [12] for decomposing $\Gamma_{k}^{(2)}$ into smaller building blocks suitable for further diagonalization. The method is based on the observation that it is sufficient to consider only a covariant constant magnetic background field in order to project the flow equation onto the present truncation.

The method consists of identifying those components of the quantum fluctuations which are appropriately oriented with respect to the background field; the latter is chosen to be of the type

$$
A_{\mu}^{a}=n^{a} \mathrm{~A}_{\mu}, \quad \mathrm{F}_{\mu \nu}=\partial_{\mu} \mathrm{A}_{\nu}-\partial_{\nu} \mathrm{A}_{\mu}=B \epsilon_{\mu \nu}^{\perp}=\text { const },
$$

where $n^{a}$ is a constant unit vector in color space, $n^{2}=1$, and $\mathrm{A}_{\mu}, \mathrm{F}_{\mu \nu}$ denote the "Abelian" gauge field and field strength. The constant tensor $\epsilon_{\mu \nu}^{\perp}$ characterizes the space directions which are affected by the constant magnetic field upon the Lorentz force, e.g., $\epsilon_{12}^{\perp}=-\epsilon_{21}^{\perp}=1$ for $B$ pointing into the 3 direction.

Let us first define two important operators involving the covariant derivative $\left(D_{\mu}[A]\right)^{a b}=\partial_{\mu} \delta^{a b}-\mathrm{i} \bar{g} A_{\mu}^{c}\left(T^{c}\right)^{a b}$ in the adjoint representation:

$$
\begin{gathered}
\left(\mathcal{D}_{\mathrm{T}}\right)_{\mu \nu}^{a b}=\left(-D^{2} \delta_{\mu \nu}+2 \mathrm{i} \bar{g} F_{\mu \nu}\right)^{a b} \\
\left(\mathcal{D}_{\mathrm{L}}\right)_{\mu \nu}^{a b}=-(D \otimes D)_{\mu \nu}^{a b} \equiv-D_{\mu}^{a c} D_{\nu}^{c b},
\end{gathered}
$$

where $\left(F_{\mu \nu}\right)^{a b}=F_{\mu \nu}^{c}\left(T^{c}\right)^{a b}$. For covariant constant fields of the type (A1) satisfying the equations of motion $\left[D_{\mu}, F_{\mu \nu}\right]$ $=0$, the operators $\mathcal{D}_{\mathrm{T}}$ and $\mathcal{D}_{\mathrm{L}}$ commute. As a consequence, projection operators can be introduced:

$$
P_{\mathrm{L}}=\mathcal{D}_{\mathrm{T}}^{-1} \mathcal{D}_{\mathrm{L}}, \quad P_{\mathrm{T}}=1-P_{\mathrm{L}},
$$

which obey $P_{\mathrm{T}, \mathrm{L}}^{2}=P_{\mathrm{T}, \mathrm{L}}, \quad P_{\mathrm{T}}+P_{\mathrm{L}}=1, \quad P_{\mathrm{T}} P_{\mathrm{L}}=0=P_{\mathrm{L}} P_{\mathrm{T}}$. The subscripts indicate that these projectors reduce to the standard longitudinal and transverse projectors in the limit $A_{\mu} \rightarrow 0$.

Another pair of projectors can be defined which act solely in color space:

$$
P_{\|}^{a b}=n^{a} n^{b}, \quad P_{\perp}^{a b}=\delta^{a b}-n^{a} n^{b} .
$$

These four projectors are remarkably efficient in the present case; differentiating our truncation for $\Gamma_{k}[A, \bar{A}]$, as given in Eq. (22), twice with respect to $A$ and the ghost fields, then setting $A=\bar{A}$ and dropping the bar, we can represent the result as 


$$
\begin{aligned}
\Gamma_{k}^{(2)}[A, A]= & P_{\mathrm{T}} P_{\perp}\left[W_{k}^{\prime} \mathcal{D}_{\mathrm{T}}\right]+P_{\mathrm{L}} P_{\perp}\left[\frac{1}{\alpha} \mathcal{D}_{\mathrm{T}}\right] \\
& +P_{\mathrm{T}} P_{\|}\left[W_{k}^{\prime}\left(-\partial^{2}\right)+W_{k}^{\prime \prime} \mathrm{S}\right] \\
& +P_{\mathrm{L}} P_{\|}\left[\frac{1}{\alpha}\left(-\partial^{2}\right)\right]+P_{\mathrm{gh}}\left[-D^{2}\right],
\end{aligned}
$$

where we introduced

$$
\mathrm{S}_{\mu \nu}=\mathrm{F}_{\mu \alpha} \mathrm{F}_{\beta \nu} \partial^{\alpha} \partial^{\beta},
$$

and $P_{\text {gh }}$ projects trivially onto the ghost sector.

Equation (A5) is perfectly suited for further manipulation, since the spectra of the operators occurring in the square brackets is known. This decomposition also offers the possibility of conveniently implementing different wave-function renormalization constants for each subcomponent.

\section{APPENDIX B: HEAT-KERNEL COMPUTATIONS}

In this appendix we summarize the results for the heatkernel traces appearing in Eq. (26). Again, it is sufficient to perform the calculation for a covariant constant background field in order to disentangle the contributions to the flow of different operators.

Let us first mention that all color traces occurring in Eq. (26) are of the form

$$
\operatorname{tr}_{\mathrm{c}} f\left(n^{c}\left(T^{c}\right)^{a b}\right)=\sum_{l=1}^{N^{2}-1} f\left(\nu_{l}\right)
$$

where $f$ is an arbitrary function, and $\nu_{l}$ denotes the eigenvalues of the matrix $\left(n^{c} T^{c}\right)^{a b}$.

We begin with the heat-kernel trace involving the Laplacian in the covariant constant magnetic background; the spectrum is given by

$$
\begin{aligned}
& \text { Spect. } D^{2}: \quad q^{2}+(2 n+1) \bar{B}_{l}, \quad \bar{B}_{l}=\bar{g}\left|\nu_{l}\right| B, \\
& n=0,1, \ldots,
\end{aligned}
$$

where $q_{\mu}$ denotes the $(d-2)$ dimensional Fourier momentum in those spacetime directions which are not affected by the magnetic field. The index $n$ labels the Landau levels; their corresponding density of states is $\bar{B}_{l} /(2 \pi)$. Tracing over the spectrum, we obtain

$$
\frac{1}{\Omega} \operatorname{Tr}_{x \mathrm{c}} \mathrm{e}^{-\lambda\left(-D^{2}\right)}=\sum_{l=1}^{N^{2}-1} \frac{2}{2(4 \pi)^{d / 2}} \frac{1}{\lambda^{d / 2}} \frac{\lambda \bar{B}_{l}}{\sinh \lambda \bar{B}_{l}}
$$

Here, $\Omega$ denotes the spacetime volume. With reference to Eq. (26), the parameter $\lambda$ can be identified with $\lambda$ $=s W_{k}^{\prime} /\left(Z_{k} k^{2}\right)$ or $\lambda=s / k^{2}$.

Next, we turn to the heat-kernel trace involving the operator $\mathcal{D}_{\mathrm{T}}$ as defined in Eq. (A2). The spectrum is given by

$$
\begin{aligned}
q^{2}+(2 n+1) \bar{B}_{l}, & \text { multiplicity }(d-2) \\
\text { Spect. } \mathcal{D}_{\mathrm{T}}: q^{2}+(2 n+3) \bar{B}_{l}, & \text { multiplicity } 1 \\
q^{2}+(2 n-1) \bar{B}_{l}, & \text { multiplicity } 1,
\end{aligned}
$$

with $q$ and $n$ as in Eq. (B2). The last line contains the Nielsen-Olesen unstable mode for $n=0$ [30], which has a tachyonic part for small momenta $q^{2}$. Tracing over the spectrum, we find

$$
\begin{aligned}
\frac{1}{\Omega} \operatorname{Tr}_{x \mathrm{cL}} \mathrm{e}^{-\lambda \mathcal{D}_{\mathrm{T}}=} & \sum_{l=1}^{N^{2}-1} \frac{2}{2(4 \pi)^{d / 2}} \frac{1}{\lambda^{d / 2}} \\
& \times\left(d \frac{\lambda \bar{B}_{l}}{\sinh \lambda \bar{B}_{l}}+4 \lambda \bar{B}_{l} \sinh \lambda \bar{B}_{l}\right) .
\end{aligned}
$$

Finally, we need the following traces:

$$
\begin{aligned}
\frac{1}{\Omega} \operatorname{Tr}_{x} \mathrm{e}^{-\lambda\left(-\partial^{2}\right)} & =\frac{2}{2(4 \pi)^{d / 2}} \frac{1}{\lambda^{d / 2}}, \\
\frac{1}{\Omega} \operatorname{Tr}_{x \mathrm{cL}} \mathrm{e}^{-\lambda\left(-\partial^{2}\right)-\lambda^{\prime} \mathrm{S}}= & \frac{2(d-1)}{2(4 \pi)^{d / 2}} \frac{1}{\lambda^{d / 2}} \\
& +\frac{2}{2(4 \pi)^{d / 2}} \frac{1}{\lambda^{d / 2}} \frac{\lambda}{\lambda+B^{2} \lambda^{\prime}},
\end{aligned}
$$

where $\mathrm{S}$ has been defined in Eq. (A6). Here and in Eq. (B5), the $\lambda$ parameters abbreviate $\lambda=s W_{k}^{\prime} /\left(Z_{k} k^{2}\right)$ and $\lambda^{\prime}$ $=s W_{k}^{\prime \prime} /\left(Z_{k} k^{2}\right)$. Equations (B3), (B5), (B6) serve as the main input for evaluating the right-hand side of the flow equation in Sec. III.

\section{APPENDIX C: EXPANSIONS}

Here we shall explicitly display the expansions which are required for the analysis of the anomalous dimension in Sec. IV. The series given below are expanded in terms of the renormalized dimensionless field strength squared $\vartheta$, but they are also related to expansions in terms of the proper time parameter $s$ or the renormalized coupling $g^{2}$. Since we are expanding an integrand and then interchange integration with expansion, the resulting series can (and will) be asymptotic, involving strongly increasing coefficients.

Neglecting all $w_{i}$ 's in the expansion of $w_{k}(\vartheta)=\vartheta$ $+w_{2}\left(\vartheta^{2} / 2\right)+w_{3}\left(\vartheta^{3} / 6\right) \ldots$, we obtain for the expansions of the auxiliary functions $f_{1,2,3}$ as defined in Eq. (28) (recall that $\left.b_{l}=\left|\nu_{l}\right| \sqrt{2 \vartheta}\right)$ : 


$$
\begin{aligned}
& \left.2 \sum_{l=1}^{N^{2}-1} f_{1}\left(s \dot{w}_{k} b_{l}\right) b_{l}^{d / 2}\right|_{w_{i} \rightarrow 0} \\
& \quad=-(d-1) \sum_{i=0}^{\infty} \frac{2^{i}\left(2^{2 i}-2\right)}{(2 i) !} \tau_{i} B_{2 i} s^{2 i-d / 2} \vartheta^{i} \\
& \quad+4 \sum_{i=0}^{\infty} \frac{2^{i}}{(2 i-1) !} \tau_{i} s^{2 i-d / 2} \vartheta^{i} \\
& 2 \sum_{l=1}^{N^{2}-1} f_{2}\left(s b_{l}\right) b_{l}^{d / 2} \\
& =-\sum_{i=0}^{\infty} \frac{2^{i}\left(2^{2 i}-2\right)}{(2 i) !} \tau_{i} B_{2 i} s^{2 i-d / 2} \vartheta^{i},
\end{aligned}
$$

where $B_{2 i}$ denotes the Bernoulli numbers, and we define $1 /(-1) !=0$. The $\tau_{i}$ are defined in Appendix E and are related to the group theoretical factors $\sum_{l=1}^{N^{2}-1}\left(\nu^{2}\right)^{i}$ that occur in the expansions given above. Whereas the expansion of $f_{3}$ vanishes in the present approximation, the expansion of its derivatives, as they occur in the last line of Eq. (31), must be retained:

$$
\begin{aligned}
& \left.\left(\partial_{t}-4 \vartheta \partial_{\vartheta}+d\right) f_{3}\left(s \dot{w}_{k}, \frac{\dot{w}_{k}}{\dot{w}_{k}+2 \vartheta \ddot{w}_{k}}\right)\right|_{w_{i} \rightarrow 0} \\
& =\sum_{i=1}^{\infty} \frac{2 i}{s^{d / 2}} \frac{\vartheta^{i}}{i !} \partial_{t} w_{i+1} .
\end{aligned}
$$

\section{APPENDIX D: CUTOFF FUNCTIONS}

In Eq. (12) we introduce the cutoff function $R_{k}(x)$ $=x r\left(x / Z_{k} k^{2}\right)$, where $r(y)$ is a dimensionless function of a dimensionless argument. For actual computations, we need the combinations $h(y)$ and $g(y)$ as well as their Laplace transforms $\tilde{h}(s)$ and $\tilde{g}(s)$ as defined in Eqs. (16) and (18).

Instead of choosing a certain cutoff function by specifying $r(y)$, we can specify a function $h(y)$, or alternatively $g(y)$, which fixes the remaining functions by virtue of Eq. (16); the direct connection between $h(y)$ and $g(y)$ can be formulated as

$$
y \frac{d}{d y} g(y)=(g(y)-1) h(y) .
$$

A similar reasoning holds for a definition of the cutoff in Laplace space by specifying one of the functions $\widetilde{h}(s)$ or $\tilde{g}(s)$, for which Eq. (D1) translates into

$$
\tilde{g}(s)+s \frac{d}{d s} \tilde{g}(s)=\tilde{h}(s)-\int_{0}^{s} d t \tilde{h}(t) \tilde{g}(s-t) .
$$

These identities can be used to define a desired cutoff in its simplest representation without the need to specify the corresponding function $r(y)$ explicitly; the latter might look very complicated. Of course, one has to take care of all the necessary conditions that a cutoff has to satisfy as listed in Eqs. (13) and (14).

During the expansion of the proper time integrand in Sec. IV, we encounter the moments of $\widetilde{h}(s)$ and $\widetilde{g}(s)$ as defined in Eq. (41). These moments can also be translated into a momentum space calculation ("y space"):

$$
\begin{gathered}
h_{-j}:=\int_{0}^{\infty} \frac{d s}{s^{j}} \widetilde{h}(s)=\frac{1}{\Gamma(j)} \int_{0}^{\infty} d y y^{j-1} h(y), \quad j>0, \\
h_{j}:=\int_{0}^{\infty} d s s^{j} \tilde{h}(s)=\lim _{y \rightarrow 0}(-1)^{j}\left(\frac{d}{d y}\right)^{(j)} h(y), \quad j \geqslant 0
\end{gathered}
$$

and equivalently for the $g_{j}$ 's.

In this work the exponential cutoff is technically advantageous; all functions involved have a simple representation:

$$
\begin{aligned}
& r(y)=\frac{1}{\mathrm{e}^{y}-1}, \quad h(y)=\frac{y}{\mathrm{e}^{y}-1}, \quad g(y)=\mathrm{e}^{-y}, \\
& \tilde{h}(s)=-\sum_{m=1}^{\infty} \delta(s-m) \frac{d}{d s}, \quad \tilde{g}(s)=\delta(s-1),
\end{aligned}
$$

where the $s$ derivative acts on the remaining propertime integrand. For the moments required in $d=4$, we find

$$
\begin{aligned}
g_{j} & =1, \\
h_{-2} & =2 \zeta(3) \simeq 2.404 \ldots, \\
h_{j} & =B_{j}, \quad j=1,2, \ldots,
\end{aligned}
$$

where $B_{j}$ symbolizes the Bernoulli numbers.

\section{APPENDIX E: SU(2) VERSUS SU(3)}

Gauge group information enters the flow equation via the color traces. In Appendix B, we evaluated these traces formally by introducing the eigenvalue of $\left(n^{c} T^{c}\right)^{a b} \rightarrow \nu_{l}, \quad l$ $=1, \ldots, N^{2}-1$. During the expansion of the right-hand side of the flow equation in Sec. IV, we encounter the following factors:

$$
\sum_{l=1}^{N^{2}-1} \nu_{l}^{2 i}=n^{a_{1}} n^{a_{2}} \ldots n^{a_{2 i}} \operatorname{tr}_{\mathrm{c}}\left[T^{\left(a_{1}\right.} T^{a_{2}} \ldots T^{\left.a_{2 i}\right)}\right]
$$

where the parentheses at the color indices denote symmetrization. For general gauge groups, these factors are not independent of the direction of $n^{a}$. Contrary to this, the left-hand side of the flow equation is a function of $\frac{1}{4} F_{\mu \nu}^{a} F_{\mu \nu}^{a} \rightarrow \frac{1}{2} B^{2}$, which is independent of $n^{a}$. Therefore, we do not need the complete factor of Eq. (E1), but only that part of the symmetric invariant tensor $\operatorname{tr}_{\mathrm{c}}\left[T^{\left(a_{1}\right.} \ldots T^{\left.a_{2 i}\right)}\right]$ which is proportional to the trivial one: 


$$
\operatorname{tr}_{\mathrm{c}}\left[T^{\left(a_{1}\right.} T^{a_{2}} \ldots T^{\left.a_{2 i}\right)}\right]=\tau_{i} \delta_{\left(a_{1} a_{2}\right.} \ldots \delta_{\left.a_{2 i-1} a_{2 i}\right)}+\cdots
$$

where we omitted further nontrivial symmetric invariant tensors. These omitted terms do not contribute to the flow of $W_{k}(\theta)$, but to the flow of other operators which do not belong to our truncation, e.g., operators involving contractions of the field strength tensor with the $d_{a b c}$ symbols.

For $\mathrm{SU}(N)$ gauge groups, we trivially deduce that

$$
\tau_{0}=N^{2}-1, \quad \tau_{1}=N \text {. }
$$

For the gauge group SU(2), all complications are absent, since there are no further symmetric invariant tensors in Eq. (E2), implying

$$
\tau_{i}^{N=2}=2, \quad i=1,2, \ldots
$$

For the gauge group $\mathrm{SU}(3)$, we do not evaluate the $\tau_{i}$ 's from Eq. (E2) directly; instead, we exploit the fact that the color unit vector can always be rotated into the Cartan subalgebra. For SU(3), we choose a color vector $n^{a}$ pointing into the 3 or 8 direction in color space, representing the two possible extremal cases:

$$
\tau_{i, 3}^{N=3}=2+\frac{1}{2^{2 i-2}}, \quad \tau_{i, 8}^{N=3}=\frac{3^{i}}{2^{2 i-2}} .
$$

Note that their limiting behavior is rather different: for $i$ $\rightarrow \infty$, we find $\tau_{i, 3}^{N=3} \rightarrow 2$, but $\tau_{i, 8}^{N=3} \rightarrow 0$.

The uncertainty introduced by the artificial $n^{a}$ dependence of the color traces is finally responsible for the uncertainty of our result for the $\mathrm{SU}(3)$ infrared fixed point.

\section{APPENDIX F: NUMERICAL COMPUTATIONS}

Since the numerical evaluation of the anomalous dimension $\eta$ depending on the coupling $G=g^{2} /\left[2(4 \pi)^{2}\right]$ as represented in Eqs. (54) and (56) is not straightforward, we mention here some details about the multidimensional integration and summation. We begin with the part $\eta_{\mathrm{a}}$ in Eq. (54): substituting $s_{1} / s_{2} \rightarrow s_{1}$, the $s_{2}$ integral can be performed, resulting in the modified Bessel function $K_{3 N^{2}-4}\left(2 \sqrt{s_{1}}\right)$. Substituting furthermore $t \rightarrow t / l$, and defining the expressions

$$
L(t):=\sum_{l=1}^{\infty} \frac{1}{2} \frac{1}{1+\cosh l t} \frac{1}{l},
$$

$$
\widetilde{K}\left(s_{1}\right):=s_{1}^{3 N^{2} / 2-1} K_{3 N^{2}-4}\left(2 \sqrt{s_{1}}\right),
$$

we obtain the representation

$$
\begin{aligned}
\eta_{\mathrm{a}}^{N=2}= & \frac{64 N G}{\Gamma\left(3 N^{2}-2\right) \pi^{2}} \int_{0}^{\infty} d t L(t) \int_{0}^{\infty} d s_{1} \widetilde{K}\left(s_{1}\right) \\
& \times\left[S\left(\frac{c G s_{1} t^{2}}{2 \pi^{4}}\right)-\frac{1}{2} S\left(\frac{c G s_{1} t^{2}}{8 \pi^{4}}\right)\right] .
\end{aligned}
$$

Apart from an easily integrable $1 / \sqrt{t}$ singularity induced by $L(t)$, the integrals are smooth and drop off exponentially for large $t$ and $s_{1}$ in the required $G$ range. The sum $S(q)$ defined in Eq. (55) converges quickly and an accuracy with an error $<1 \%$ requires only $\mathcal{O}(100)$ terms or less. The sum $L(t)$ is rather slowly converging for small $t$, but the same accuracy can be obtained by including $\mathcal{O}\left(10^{5}-10^{6}\right)$ terms. Depending on the actual value of the arguments $t$ and $q$, we adjust the included number of terms dynamically.

For the part $\eta_{\mathrm{b}}$, different complications occur. Beginning with Eq. (56), we substitute $s \rightarrow s t \sqrt{c G /\left(2 \pi^{2}\right)}$ (and similarly for $s^{\prime}$ ) and find

$$
\begin{aligned}
\eta_{\mathrm{b}}^{N=2}= & -\frac{32 N G}{\Gamma\left(3 N^{2}-2\right)} \operatorname{Re} \int_{0}^{\infty} \frac{(1+\mathrm{i})}{\sqrt{2}} d t \frac{\mathrm{e}^{[(1+\mathrm{i}) / \sqrt{2} t]}}{\left(\mathrm{e}^{[(1+\mathrm{i}) / \sqrt{2} t]}+1\right)^{2}} \\
& \times I_{s}\left(\sqrt{\frac{c G}{2 \pi^{2}} t}\right),
\end{aligned}
$$

where we defined

$$
\begin{aligned}
I_{s}(x)= & \frac{1}{x} \int_{0}^{x} d s\left(\frac{d}{d s}\right)^{\left(3 N^{2}-3\right)} \\
& \times\left.\frac{d}{d s^{\prime}} s^{3 N^{2}-3} s^{\prime} S\left(-\mathrm{i} s s^{\prime}\right)\right|_{s^{\prime}=x-s} .
\end{aligned}
$$

The problem here is that the derivatives cannot be carried out numerically with a sufficient accuracy, but have to be computed analytically within the sum representation for $S$ $\left(-\mathrm{i} s s^{\prime}\right)$. This implies that each term in the sum then consists of $\sim 20$ terms for $\mathrm{SU}(2)$ and $\sim 50$ for $\mathrm{SU}(3)$. This limits the generalization of the calculation to higher gauge groups for technical reasons. The remaining $s$ and $t$ integrations can easily be performed to a high accuracy. We estimate the total error of the numerical computation to be within a few percent.
[1] F. J. Wegner and A. Houghton, Phys. Rev. A 8, 401 (1973); K. G. Wilson and J. B. Kogut, Phys. Rep. 12, 75 (1974); S. Weinberg, in the Proceedings of the 1976 International School of Subnuclear Physics, Erice, 1976 (Plenum, New York, 1976), p. 1; J. Polchinski, Nucl. Phys. B231, 269 (1984); A. Hasenfratz and P. Hasenfratz, ibid. B270, 687 (1986); Helv. Phys. Acta 59, 833 (1986).

[2] C. Wetterich, Phys. Lett. B 301, 90 (1993); Nucl. Phys. B352,
529 (1991)

[3] M. Bonini, M. D’Attanasio, and G. Marchesini, Nucl. Phys. B421, 429 (1994).

[4] U. Ellwanger, Phys. Lett. B 335, 364 (1994).

[5] M. Reuter and C. Wetterich, Nucl. Phys. B417, 181 (1994).

[6] M. Bonini, M. D’Attanasio, and G. Marchesini, Nucl. Phys. B437, 163 (1995); Phys. Lett. B 346, 87 (1995).

[7] U. Ellwanger, M. Hirsch, and A. Weber, Z. Phys. C 69, 687 
(1996); Eur. Phys. J. C 1, 563 (1998).

[8] M. D’Attanasio and T. R. Morris, Phys. Lett. B 378, 213 (1996).

[9] D. F. Litim and J. M. Pawlowski, in The Exact Renormalization Group, Proceedings of the Workshop, Faro, Portugal, edited by A. Krasnitz et al. (World Scientific, Singapore, 1999).

[10] T. R. Morris, Nucl. Phys. B573, 97 (2000).

[11] T. R. Morris, J. High Energy Phys. 12, 012 (2000); S. Arnone, Y. A. Kubyshin, T. R. Morris, and J. F. Tighe, Int. J. Mod. Phys. A 16, 1989 (2001).

[12] M. Reuter and C. Wetterich, Phys. Rev. D 56, 7893 (1997).

[13] U. Ellwanger, Nucl. Phys. B560, 587 (1999); Eur. Phys. J. C 7, 673 (1999); Nucl. Phys. B531, 593 (1998).

[14] F. Freire, Phys. Lett. B 526, 405 (2002).

[15] J. I. Latorre and T. R. Morris, J. High Energy Phys. 11, 004 (2000).

[16] H. Gies and C. Wetterich, Phys. Rev. D 65, 065001 (2002).

[17] D. F. Litim, Phys. Lett. B 486, 92 (2000); Phys. Rev. D 64, 105007 (2001).

[18] D. F. Litim, J. High Energy Phys. 11, 059 (2001).

[19] F. Freire, D. F. Litim, and J. M. Pawlowski, Phys. Lett. B 495, 256 (2000).

[20] L. F. Abbott, Nucl. Phys. B185, 189 (1981); W. Dittrich and M. Reuter, Selected Topics in Gauge Theories, Lecture Notes in Physics Vol. 244 (Springer, Berlin, 1986), p. 1.

[21] F. Freire and C. Wetterich, Phys. Lett. B 380, 337 (1996).

[22] S. B. Liao, Phys. Rev. D 53, 2020 (1996).

[23] S. B. Liao, Phys. Rev. D 56, 5008 (1997); R. Floreanini and R. Percacci, Phys. Lett. B 356, 205 (1995); B. J. Schaefer and H. J. Pirner, Nucl. Phys. A660, 439 (1999); A. Bonanno and D. Zappala, Phys. Lett. B 504, 181 (2001).

[24] D. F. Litim and J. M. Pawlowski, Phys. Lett. B 516, 197 (2001); Phys. Rev. D 65, 081701(R) (2002).

[25] D. F. Litim and J. M. Pawlowski, hep-th/0202188.

[26] G. K. Savvidy, Phys. Lett. 71B, 133 (1977).

[27] J. M. Pawlowski, Int. J. Mod. Phys. A 16, 2105 (2001).

[28] D. F. Litim and J. M. Pawlowski, hep-th/0203005.

[29] D. F. Litim and J. M. Pawlowski, Phys. Lett. B 435, 181 (1998); O. Lauscher and M. Reuter, Phys. Rev. D 65, 025013 (2002).

[30] N. K. Nielsen and P. Olesen, Nucl. Phys. B144, 376 (1978).

[31] M. Bonini, G. Marchesini, and M. Simionato, Nucl. Phys. B483, 475 (1997).
[32] G. Hardy, Divergent Series (Oxford University Press, Oxford, England, 1949); C. M. Bender and S. A. Orszag, Advanced Mathematical Methods for Scientists and Engineers (McGrawHill, New York, 1978).

[33] J. C. Le Guillou and J. Zinn-Justin, Large Order Behavior Of Perturbation Theory (North-Holland, Amsterdam, 1990); G. V. Dunne and T. M. Hall, Phys. Rev. D 60, 065002 (1999); G. V. Dunne and C. Schubert, Nucl. Phys. B564, 591 (2000).

[34] I. S. Gradshteyn and I. M. Ryzhik, Table of Integrals, Series, and Products, 6th ed., edited by Alan Jeffrey (Academic Press, San Diego, 2000).

[35] B. Bergerhoff and C. Wetterich, Phys. Rev. D 57, 1591 (1998).

[36] M. Luscher, R. Sommer, P. Weisz, and U. Wolff, Nucl. Phys. B413, 481 (1994).

[37] Y. L. Dokshitzer, A. Lucenti, G. Marchesini, and G. P. Salam, J. High Energy Phys. 05, 003 (1998); Y. L. Dokshitzer, hep-ph/9812252.

[38] E. Eichten et al., Phys. Rev. Lett. 34, 369 (1975); 36, 1276(E) (1976); T. Barnes, F. E. Close, and S. Monaghan, Nucl. Phys. B198, 380 (1982); S. Godfrey and N. Isgur, Phys. Rev. D 32, 189 (1985).

[39] A. C. Mattingly and P. M. Stevenson, Phys. Rev. Lett. 69, 1320 (1992).

[40] T. Banks and A. Zaks, Nucl. Phys. B196, 189 (1982); G. Grunberg, Phys. Rev. D 65, 021701(R) (2002); E. Gardi and G. Grunberg, J. High Energy Phys. 03, 024 (1999).

[41] D. V. Shirkov and I. L. Solovtsov, Phys. Rev. Lett. 79, 1209 (1997); Teor. Mat. Fiz. 120, 482 (1999) [Theor. Math. Phys. 120, 1220 (1999)].

[42] N. G. Stefanis, W. Schroers, and H. C. Kim, Eur. Phys. J. C 18, 137 (2000); D. V. Shirkov, ibid. 22, 331 (2001).

[43] L. von Smekal, R. Alkofer, and A. Hauck, Phys. Rev. Lett. 79, 3591 (1997); Ann. Phys. (N.Y.) 267, 1 (1998); 269, 182(E) (1998); D. Atkinson and J. C. Bloch, Phys. Rev. D 58, 094036 (1998); D. Zwanziger, ibid. 65, 094039 (2002); C. Lerche and L. von Smekal, ibid. 65, 125006 (2002); C. S. Fischer and R. Alkofer, hep-ph/0202202.

[44] F. D. Bonnet, P. O. Bowman, D. B. Leinweber, A. G. Williams, and J. M. Zanotti, Phys. Rev. D 64, 034501 (2001); K. Langfeld, H. Reinhardt, and J. Gattnar, Nucl. Phys. B621, 131 (2002); K. Langfeld, talk delivered at NATO workshop on "Confinement, Topology and other Nonperturbative Aspects of QCD,” Stará Lesná, Slovakia, 2002. 